\title{
Testing a Conjecture On Quantum Electrodynamics
}

\author{
Christoph Schiller*
}

\begin{abstract}
A geometric Planck-scale model of quantum electrodynamics is tested against observations. Based on Dirac's proposal to describe spin 1/2 particles as tethered objects, elementary fermions are conjectured to be fluctuating rational tangles with unobservable tethers. As Battey-Pratt and Racey showed, when such tangles propagate, they obey the free Dirac equation.

Classifying rational tangles yields the observed spectrum of elementary fermions. Classifying deformations of tangle cores yields exactly three types of gauge interactions. They exchange three types of elementary gauge bosons and have the symmetry groups $\mathrm{U}(1)$, broken $\mathrm{SU}(2)$ and $\mathrm{SU}(3)$. The possible rational tangles for fermions, Higgs and gauge bosons allow only the observed Feynman diagrams. The complete Lagrangian of the standard model arises, including the Lagrangian of quantum electrodynamics.

Over 50 tests of the tangle model are deduced. They include details on the perturbation expansion of the g-factor and the existence of limit values for electric and magnetic fields. Measurements agree with most tests, though this could change in the future. Some tests are genuine predictions that still need to be checked.

In particular, the geometry of the process occurring at QED interaction vertices suggests an ab-initio estimate for the fine structure constant. In addition, the average geometric shapes of the elementary particle tangles suggest ab-initio lower and upper limits for the mass values of the electron and the other leptons.
\end{abstract}

Keywords: quantum electrodynamics; electron mass; fine structure constant; strand conjecture; tangle model.

Motion Mountain Research, Munich, Germany. ORCID 0000-0002-8188-6282, cs@motionmountain.net 


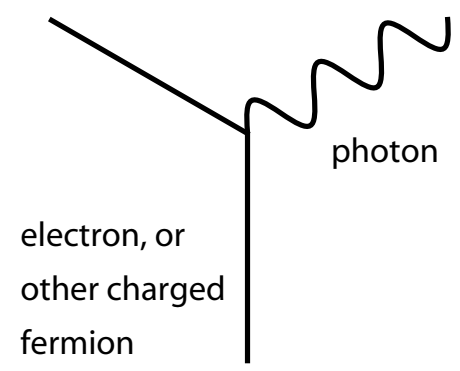

Figure 1: The two questions of quantum electrodynamics: What happens at the interaction vertex? What happens during the propagation of electrons and photons?

\section{The open issues of quantum electrodynamics}

After the successful development of quantum electrodynamics in the middle of the twentieth century, two questions about the origin of its fundamental constants remain:

$\triangleright$ What determines the full propagator of the electron and thus its mass value?

$\triangleright$ What exactly happens at the fundamental interaction vertex of quantum electrodynamics and how does this process determine the fine structure constant?

Answering these questions is necessary, for example, to understand fully the wavelength of atomic spectral lines, the colours of nature, and the size of atoms. The answers also determine most of material science, of chemistry and of biology. Equivalently, one can ask: what happens when a charged particle emits or absorbs a photon? The details of the process, usually illustrated with the Feynman diagram of Figure 1, are hidden in the point-like vertex.

In the past, most attempted explanations of the fine structure constant have been guesses [1, [2, 3], and thus not satisfactory. These attempts failed because they did not correctly describe the process of photon absorption and emission.

The same situation holds for the electron mass value. The value of the inertial mass determines the propagator of the electron. But the details of propagation are hidden in the propagator lines of the Feynman diagram. Past attempts to explain mass values of elementary particles failed because they did not correctly describe particle propagation.

A further challenge arises. Any proposed explanation of the fine structure or the electron mass can only be correct if the explanation also derives the particle spectrum and all the other unexplained fundamental constants of the standard model of particle physics: the nuclear coupling constants, the other particle masses, and the mixing angles. The attempt by Glashow and Nanopoulos [4] was only a partial explanation, and therefore an unsuccessful one. In the past one hundred years, no proposal that describes all gauge interactions and all elementary particles has provided any hope that an explanation for the fundamental constants is within reach. Instead, practically all such proposals predicted new effects or new particles beyond the standard model that failed to show up [5]. 
The present paper explores a candidate for a unified description of quantum phenomena with six central properties: the candidate model has a simple foundation, describes propagation, describes interaction vertices, agrees with experimental data, predicts no physics beyond the standard model, and allows calculating the fundamental constants. The emphasis of the following exploration is on the tests and implications for quantum electrodynamics and for its two fundamental constants.

The starting point is the recently proposed strand conjecture [6]. The conjecture posits that particles, space and horizons are made of fluctuating and unobservable strands of Planck length radius. Only strand crossings that change orientation, so-called skew crossing switches, are observable; the reason will become clear below. Elementary particles are modelled as unknotted, so-called rational tangles that spin. Interactions are modelled as deformations of elementary particle tangles.

As summarized below, the strand conjecture implies the four fundamental interactions, yields the $\mathrm{U}(1)$, broken $\mathrm{SU}(2)$ and $\mathrm{SU}(3)$ gauge symmetries of the gauge interactions, and produces the observed spectrum of elementary bosons and fermions. It appears that the strand conjecture reproduces the full Lagrangian of the standard model of particle physics, with massive neutrinos and PMNS mixing [6,7]. In the microscopic domain, the conjecture predicts that the standard model with massive neutrinos is valid at all measurable energies, without any modification. The conjecture thus predicts the lack of new measurable effects beyond the standard model, of any kind whatsoever.

In addition to the agreement with experiment, strands imply the possibility to calculate the fundamental constants of nature, in particular the fine structure constant and the mass values of the electron and of the other leptons. This possibility is explored below. The remaining coupling constants and the other elementary particle masses, as well as the various mixing angles and phases, were briefly investigated in references [6], [7] and [8]. To make the present paper selfcontained, the first sections summarize the strand conjecture. Throughout the text, a thorough list of predictions and experiments to test the conjecture in the domain of quantum electrodynamics is provided.

\section{The origin of the conjecture}

Bohr used to present quantum theory as consequence of a smallest observable action value $\hbar$ [9]. Dirac then included the maximum energy speed $c$ into quantum theory. From around 1929 onwards, Dirac mentioned the so-called string trick or belt trick in his lectures. Illustrated in Figure 2 the trick describes the main properties of spin $1 / 2$ as the result of tethered rotation. As Dirac wrote [10], the trick also explains that no spin value below $\hbar / 2$ is possible. A smallest angular momentum $\hbar / 2$ still implies a smallest observable action value $\hbar$.

In nature, spin $1 / 2$ is observed, but particle tethers are not. Dirac's tether proposal provided a first hint that nature might be described with extended constituents that are themselves unobservable, but whose crossing switches are observable. In 1980, Battey-Pratt and Racey went further: 


\section{The belt trick or string trick:}

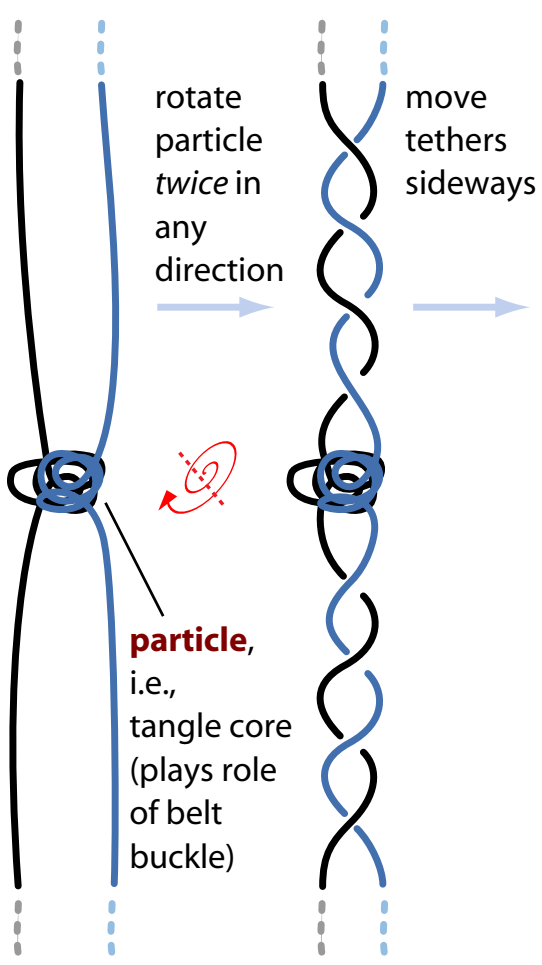

double tethered rotation is no rotation

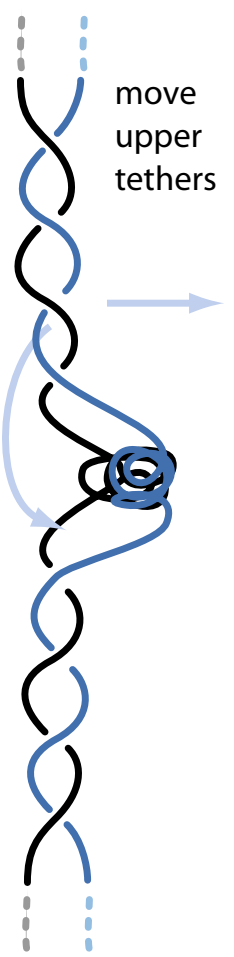

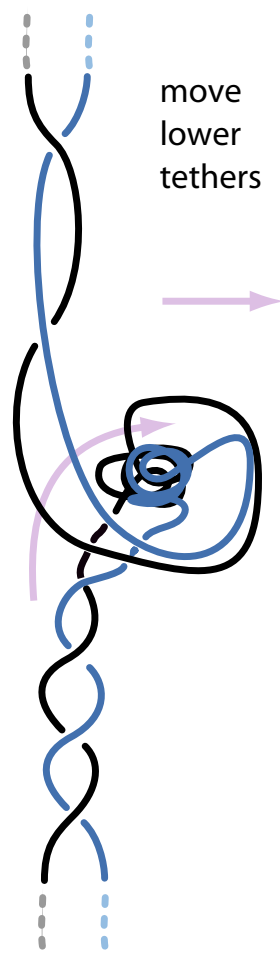

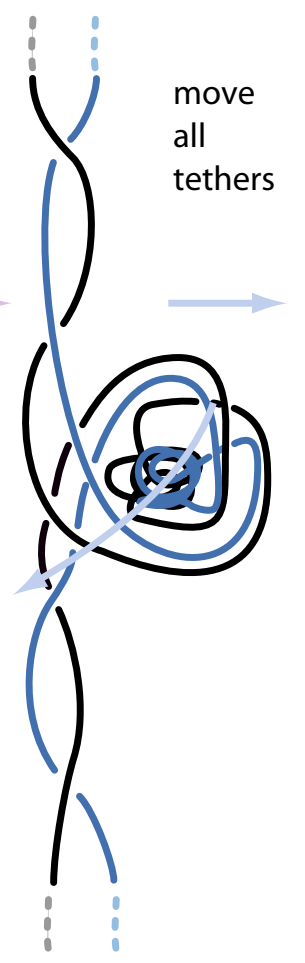

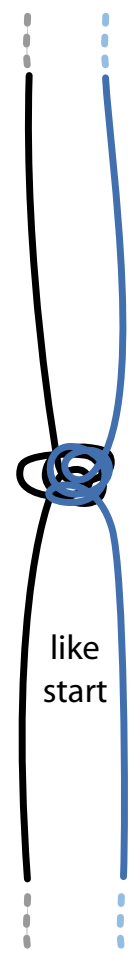

Resulting observation: probability density and phase for unobservable tethers with observable crossings
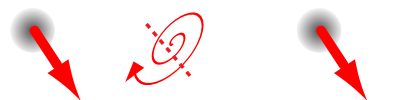

phase

Figure 2: The belt trick or string trick: a double rotation, thus by $4 \pi$, of a tethered particle, such as a belt buckle or a tangle, is equivalent to no rotation - if the tethers are allowed to fluctuate and untangle as shown. Untangling tethers is not possible after a particle rotation by only $2 \pi$ : tangles with 4 or more tethers thus show the properties of spin $1 / 2$ particles. As a result, a tethered particle is able to rotate continuously. In the strand conjecture, the trick also couples rotation and displacement of the tangle core. The coupling of core rotation frequency and core displacement determines particle mass, as argued in Section 20, (Modified from reference [7].)

they showed that the full free Dirac equation could be deduced from unobservable tethers attached to localized masses [11]. They showed that Dirac's trick implies Dirac's equation. The result suggests that every quantum effect can be thought as being due to unobservable extended constituents with observable crossing switches.

A further foundation of the strand conjecture is the realization that not only quantum theory and special relativity, but also general relativity is based on a limit statement, namely on the maximum force $c^{4} / 4 G[12,13,14,15,16]$. This result is valid independently of the strand conjecture. Combining the three limits $c, \hbar$ and $c^{4} / 4 G$ yields a minimum length and a minimum time. All 


\section{The fundamental Planck-scale principle of the strand conjecture}

Strand conjecture description
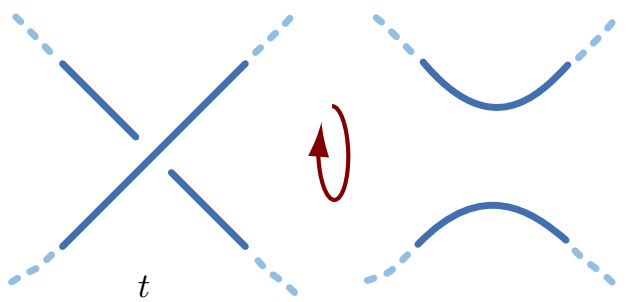
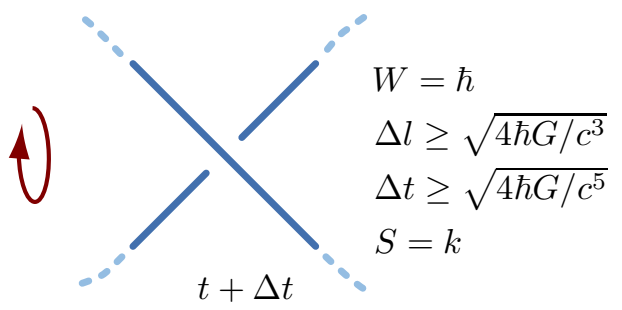

Resulting

observation

A fundamental event localized

in space

Figure 3: The fundamental principle of the strand conjecture describes the simplest observation possible in nature, a fundamental event. In the strand conjecture, a fundamental event is almost pointlike. A fundamental event results from a skew strand crossing switch - the exchange of underpass and overpass - at a location in three-dimensional space. The strands themselves are not observable, are impenetrable, and are best imagined as ropes or cables having Planck-size radius. The crossing switch defines $\hbar$ as the unit of the physical action $W$. Both the Planck length and the Planck time arise, respectively, from the smallest and from the fastest possible crossing switch. The fastest crossing switch is discussed in Section 14 The crossing (switch) can also be taken as the strand realization of a qubit.

these invariant limits are incorporated in the fundamental principle of the strand conjecture.

\section{The strand conjecture and its fundamental principle}

The strand conjecture states that everything in nature - matter, radiation, space, and horizons - is made of strands that fluctuate at the Planck scale $[6,7]$.

$\triangleright$ A strand is defined as smooth simple curved line - a one-dimensional, open, continuous, everywhere infinitely differentiable subset of $\mathbb{R}^{3}$ or of a curved 3-dimensional Riemannian space, with trivial topology and without endpoints - that is surrounded by a perpendicular disk of Planck radius $\sqrt{\hbar G / c^{3}}$ at each point of the line, and whose shape is randomly fluctuating over time.

Strands thus resemble thin flexible ropes or cables with a Planck-size radius - with the property that they cannot be cut, and thus that they are not made of parts. From the viewpoint of mathematics, the definition of strands extends the usual definition that is used in knot theory for ropelength calculations [17, 18] by including shape fluctuations. The definition implies that strands cannot interpenetrate or intersect. From the physics viewpoint, strands have no observable properties. But even though strands are not observable, their topological tangling is, as will become clear shortly.

With their tiny, but finite radius, strands visualize the minimum length $\sqrt{4 \hbar G / c^{3}}$ as the shortest distance between two strand segments. When physical observables are introduced, as explained below, strands imply that the minimum length is an unattainable limit. 
The strand conjecture claims:

$\triangleright$ Crossing switches - the exchange of underpass and overpass - determine the Planck units, and in particular $\hbar$, as illustrated in Figure 3,

$\triangleright$ Although strands are themselves unobservable, crossing switches are observable, because of their relation to $\hbar, c, k$ and $G$.

$\triangleright$ Physical space is a network of strands. Horizons are weaves of strands. Particles are tangles of strands.

$\triangleright$ Physical motion minimizes the number of observable crossing switches of unobservable fluctuating strands.

The first statement is called the fundamental principle. The other three statements follow from the first. In simple terms, the strand conjecture claims that Figure 3 contains all of physics. In particular, the strand conjecture claims that the figure contains quantum electrodynamics. This is argued in the following.

The term crossing always implies a skew crossing or apparent crossing, when drawn in two dimensions: a strand crossing always consists of a strand overpass and a strand underpass. In three dimensions, strands are thus always at a distance, as illustrated in Figure 3 and Figure 4 . In particular, crossing switches - the exchange of underpass and overpass - always arise via strand deformations, and in no other way. The complete reason that crossing switches are observable and that nothing else is - is given below, in section 14 .

A crossing switch defines a physical event. In the strand conjecture, events are processes. The crossing switch is the most fundamental event and the most fundamental process. All observed processes in nature are composed of crossing switches; this includes macroscopic and microscopic motion of matter and radiation, gauge interactions, gravitational field evolution, gravitation waves, and every measurement.

In summary, all physical observables - such as length, mass or field intensities - emerge from combinations of crossing switches. This possible because every measurement is a process, and because crossing switches define all physical units. Continuous observables arise through the averaging of fluctuations. Particles properties, such as quantum numbers, quark masses or coupling constants, arise through a combination of topological and geometric effects, as shown below.

\section{Wave functions and particles}

Crossing switches occur at crossings.

$\triangleright$ Crossings allow to define wave functions.

As illustrated in Figure 4, a skew strand crossing has the same properties that characterize a wave function: the geometry of crossings allows defining density, position, orientation, and phase. (The density is given by the inverse minimum distance; position is the midpoint of the shortest distance 


\section{Strand crossings have the same properties as wave functions}

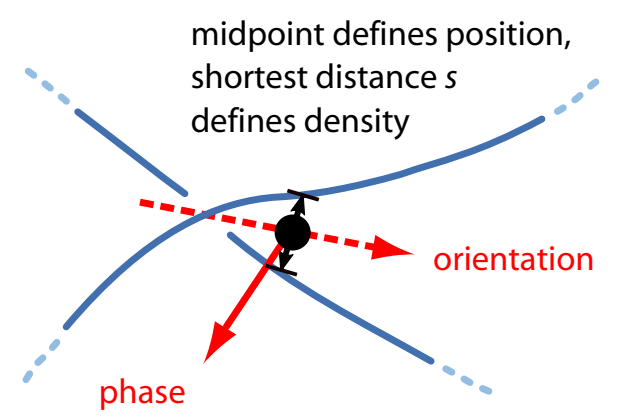

Figure 4: The geometric properties of a skew strand crossing - a strand overpass and a strand underpass separated by a shortest distance $s$-resemble those of wave functions. Both crossings and wave functions allow defining position, orientation, phase and density. For both, the absolute phase value around the orientation axis can be chosen freely. In contrast, for both, phase differences due to rotations around the axis of orientation are always uniquely defined.

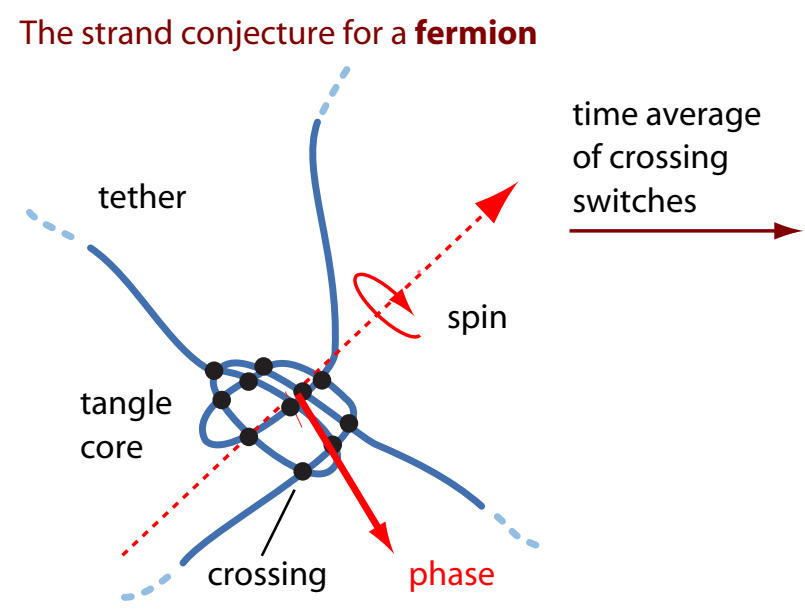

Observation

Figure 5: In the strand conjecture, the wave function is due to fluctuating crossings, and the probability density is due to fluctuating crossing switches - both after averaging. The phase of the wave function arises as the vector sum of all the crossing phases. Wave functions due to strand crossings form a Hilbert space. The tethers - strands that continue up to large spatial distances - lead to spin $1 / 2$ behaviour under rotations and to fermion behaviour under particle exchange. The tethers imply that tangle core rotation and core displacement are related; this allows defining a mass value. The relation between core rotation and core displacement also implies that fermion tangle cores move more slowly than light.

segment $s$; orientation and phase are defined with suitable cross products and sums of the vector representing $s$ and of the two unit tangent vectors of the strands at the endpoints of $s$.) Geometrically, a crossing is described by one real number, describing the minimum strand distance or density, and by four angles defining the crossing geometry around the position of the crossing. 
The geometric parameters of a crossing can be mapped to the parameters of the Pauli wave function - or to (half of) those of a Dirac wave function. In particular, the phase of the wave function of a particle arises as the sum of all crossing phases in the particle tangle, averaged over the fluctuations. The freedom in the definition of the absolute phase value is at the origin of the freedom of gauge choice.

In the strand conjecture, all continuous observables arise through time averaging.

$\triangleright$ The averaging time is a few Planck times long.

Averaging is so fast that it has no other influence on observations. Wave functions, local field intensities and even space itself [8] arise through time averaging of crossing fluctuations.

In the strand conjecture, all elementary fermions are rational tangles, i.e., open tangles that are unknotted. Only rational tangles - tangles that arise by switching tethers around in space by braiding them - allow reproducing the particle transformations that occur in interactions. No other topological structure is able to reproduce this: neither knots, nor links, nor prime tangles, nor virtual knots, nor loops, nor actual crossings, nor graphs. Equivalently,

$\triangleright$ Elementary fermions are made of unknotted but tangled tethers.

For a tangle of fluctuating strands, the average crossing distribution is the wave function, and the average crossing switch distribution is the probability distribution. The connection is illustrated in Figure 5. For a particle tangle, the average phase, the average density, and the two average spin orientation angles define the (first) two complex components of the Dirac wave function $\psi$ for a particle. For the mirror tangle, the corresponding averages define the (last) two complex components of $\psi$, for the antiparticle.

In his lectures, Dirac used a system equivalent to that of Figure 2 to demonstrate that a single tethered tangle core behaves, under rotations, like a spin $1 / 2$ particle. Indeed, a double rotation of a tethered core can be undone by rearranging the tethers only; in contrast, this is impossible after a single rotation. Dirac's demonstration can be extended to show that two tethered cores also imply that under exchange, tangle cores behave as fermions. Indeed a double exchange of tethered cores can be undone by rearranging the tethers only; in contrast, this is impossible after a single exchange. Both results apply independently of the number of tethers, as long as their number is 3 or larger. Videos that visualize both spin $1 / 2$ and fermion behaviour exist on the internet, produced by Martos [19].

In summary, tethered particles reproduce spin as core rotation and particle exchange as core exchange. Their crossing fluctuations reproduce wave functions. The suspicion that every quantum motion - also translation, scattering and interactions - can be described with tethered particles turns out to be correct.

\section{From tethers to the free Dirac equation}

This section summarizes how to deduce the Dirac equation from strands. 
In 1980, Battey-Pratt and Racey showed [11] that every tethered massive quantum particle thus every little massive sphere with attached strands that leave up to spatial infinity - is described by the Dirac equation for free particles. In other terms, Battey-Pratt and Racey assumed unobservable strands attached to a central mass and derived the Dirac equation. They even wrote Dirac about it, but they got no answer. Unfortunately, Dirac passed away shortly afterwards.

In the strand conjecture, the result of Battey-Pratt and Racey is extended. The massive particle itself is also assumed to be made of strands: an elementary fermion is conjectured to be a (rational) tangle core, i.e., the tangled region of a tangle whose tethers reach up to large distances. (When strands are imagined as ropes or cables that are pulled at the ends or at infinity, the tangle core is the region containing curved strands.) The particle tangle defines the 4-component spinor $\psi(x)$ in the following way (in the usual representation):

$\triangleright$ Averaged over a few Planck times, the position of the center of the core yields the maximum of the probability density.

$\triangleright$ At each position $x$, the upper two components of the spinor $\psi(x)$ are defined by the local average of finding, at that position, a tangle with a specific orientation and phase.

$\triangleright$ At each position $x$, the lower two components of the spinor $\psi(x)$ are defined by the local average of finding, at that position, a mirror tangle with a specific orientation and phase.

In the strand conjecture, a fermion moving freely through space can thus described by a constantly rotating tangle core whose central position is advancing through space. The free motion of a tangle thus models Feynman's description of a quantum particle as an advancing and rotating arrow [20]: the arrow is the phase of the tangle. Strands also visualize the description of Hestenes $[21,22,23]$ of the Dirac equation. The free motion of a fermion implies that advancing tangles with rotating cores are a model for fermion propagators. The relation between rotational and translational motion defines the inertial mass of the particle.

In the strand conjecture, a fermion moving freely through space can also be described with the help of a wave function and a probability density: the spatial region of maximum density, i.e., the region with most crossing switches, advances, and at the same time, the phase rotates in space. In the tangle model, the faster the particle tangle rotates and advances, the more its spin is aligned with momentum. Even at the highest rotation frequency possible (the corrected Planck frequency), the translational motion of a tangle is smaller than the speed of light $c$. Lorentz covariance is ensured. In short, particle tangles do behave like fermion propagators. And indeed, as Battey-Pratt and Racey showed, tethered relativistic particles are described by the free Dirac equation.

In the strand conjecture, strands are not observable, but their crossing switches are. Using the fundamental principle, the result [11] of Battey-Pratt and Racey can be rephrased in the following concise way:

$\triangleright$ The free Dirac equation is essentially a differential version of Dirac's string trick, or belt trick. 
Another way to express a central aspect of the connection between the belt trick and the Dirac equation is the following:

$\triangleright$ The belt trick implies the $\gamma^{\mu}$ matrices and their Clifford algebra, i.e., their geometric algebra properties [21, 22, 23].

$\triangleright$ The first two components of the $\gamma^{\mu}$ matrices describe their effect on the tangle core, i.e., on the particle.

$\triangleright$ The last two components of the $\gamma^{\mu}$ matrices describe their effect on the mirror tangle core, i.e., on the antiparticle.

One notes that with the tangles given below for each elementary particle, particles and antiparticles can be transformed into each other by moving certain tethers with respect to the others. This is only possible with rational tangles, as illustrated below, in Figure 9 .

A second, equivalent way to understand the appearance of the free Dirac equation from strands is the following. The free Dirac equation

$$
i \hbar \gamma^{\mu} \partial_{\mu} \psi=m c \psi
$$

arises from five basic properties:

1. The action limit given by $\hbar$, which yields wave functions $\psi$,

2. The energy speed limit for massive particles given by $c$, which yields Lorentz transformations and invariance,

3. The spin $1 / 2$ properties in Minkowski space-time,

4. Particle-antiparticle symmetry, this and the previous point being described by the $\gamma^{\mu}$ matrices,

5. A particle mass value $m$ that connects phase rotation frequency and wavelength using the imaginary unit $i$.

These five properties are necessary and sufficient to yield the free Dirac equation. (The connection between the $\gamma^{\mu}$ matrices and the geometry of spin was first made about a century ago by Fock and Iwanenko [24].) The tangle model of particles reproduces these five properties in the following way:

1. All observables are due to crossing switches, which imply a minimum observable action $\hbar$ (see Figure 3) and the existence of a wave function (see Figure 4 and Figure 5),

2. Tangle cores are constrained to advance less than one Planck length per Planck time, thus less than $c$ (see Figure 2),

3. Tangle core rotation connects rotation and displacement and generates a finite mass value $m$ much smaller than the Planck mass (see Figure 2 and Section 19), 
4. Tethering reproduces the spin $1 / 2$ properties for rotation, exchange and boosts, and thus introduces the $\gamma^{\mu}$ matrices (see Figure 2), with tangle and mirror tangle corresponding to particle and antiparticle,

5. Tangle rotation through the belt trick corresponding to particle propagation.

Both in nature and in the strand conjecture, the inability to observe action values below $\hbar$ leads to wave functions and probability densities. Both in nature and in the strand conjecture, the inability to observe speed values larger that $c$ leads to Lorentz invariance and the relativistic energymomentum relation. Both in nature and in the strand conjecture, together with the mass and the spin $1 / 2$ properties due to tethers, the $\gamma^{\mu}$ matrices and the Dirac equation for a free particle arise, as explained by Simulik [25, 26, 27]. Electromagnetic fields will be included below, in Section (12). Exactly like usual quantum theory, also the tangle model implies probabilities, Zitterbewegung, interference, a Hilbert space, contextuality, entanglement, mixing, decoherence all other quantum effects, as shown in detail elsewhere [28].

The strand conjecture also explains the existence of quantum motion in a third, more general way. Modern physics has shown that all motion can be described with the principle of least action. When the leat action principle is applied, the Lagrangian describes how to determine the value of the action. Now, in the strand conjecture, action is the number of crossing switches. The principle of least action then simply becomes the principle of fewest crossing switches. In modern physics, the usual statement is: motion minimizes action. In the strand conjecture, the corresponding statement is: motion minimizes crossing switches. For fermions, after suitable spatial averaging, this general idea leads to the free Dirac Lagrangian and to the free Dirac equation.

A fourth argument for the validity of the Dirac equation uses the derivation by Lerner [29], which is based on two properties. First, the definition of spin using strands implies that the spin current is conserved. Second, the definition of spin using strands also implies the Lorentz covariance of spin, i.e., the proper behaviour under rotations and boosts. (This second property is also shown explicitly by Battey-Pratt and Racey [11].) Together, as Lerner showed, these two properties imply the Dirac equation. Both properties are reproduced by the tangle model. In particular, Lorentz covariance arises because under boosts, strands change shape and their crossing density the resulting wave function - transform as expected.

In summary, strands deduce the Dirac equation from the fundamental principle. More precisely, the Dirac equation results from the behaviour of crossings in fluctuating rational tangles. In particular, the usual expressions for the usual fermion propagator follow from strands. In short, strands visualize how the quantum of action $\hbar$ leads to the free Dirac equation.

\section{Predictions about relativistic quantum theory}

Strands predict the lack of even the tiniest deviation from relativistic quantum theory. The slightest deviation from the free Dirac equation would falsify the strand conjecture. The predictions remains valid when gauge interactions are included, as shown below. Strands yield no deviations at any 
measurable energy - strands just yield small effects at the Planck scale, when gravity comes into play.

Test 1: Finding a situation or an energy scale for which the Dirac equation is not valid would falsify the strand conjecture.

In addition to the Dirac equation, the fundamental principle of the strand conjecture implies that every Planck unit (corrected by changing $G$ to $4 G$ ) is an insurmountable local limit to physical observables in the quantum domain. More precisely, the strand conjecture predicts the lack of any trans-Planckian effects. Therefore,

Test 2: Observing an elementary particle whose energy is larger than $\sqrt{\hbar c^{5} / 4 G}=6.1 \cdot 10^{18} \mathrm{GeV}$ would falsify the strand conjecture. The same applies to Planck limits for momentum, distance, action, temperature, force, power, etc.

The tangle model implies that elementary fermions, such as the electron, have an effective size of the order of the Compton wavelength that explains their wave properties and spin properties. (At the same time, elementary fermions are not always rigidly rotating objects, as explored below.) Furthermore, as shown in Section 11, charged elementary fermions are effectively point particles when probed by electromagnetic fields. The tangle model thus realizes both apparently contradictory requirements, spelled out by Barut [30], for a complete description of elementary particles.

Test 3: Observing an elementary particle with a cross section larger than $O(1)$ times the Planck area would falsify the tangle model.

Test 4: Observing an elementary particle with a spin that is not an integer multiple of $\hbar / 2$ would falsify the tangle model.

In summary, the description of relativistic quantum theory with strands is identical to the usual one. Nevertheless, strands imply one new result. If elementary particles are rational tangles, then their spectrum, their interactions, their quantum numbers, their masses and all their other particle properties are not free, but are fixed by their tangle structure. For example, mass is given by the frequency of the spontaneous belt trick. This new result can be checked.

\section{Predictions about the spectrum of elementary particles}

This section summarizes how strands lead to the observed spectrum of bosons and fermions. The details were already explored elsewhere [6, 7].

Elementary bosons can consist of one, two or three strands. More strands imply composite systems. The Reidemeister moves yield the known gauge groups (as explained below) and thus suggest that one-stranded bosons correspond to photons, two-stranded bosons to the $W_{1}, W_{2}$ or $W_{3}$, and three-stranded bosons to gluons. After symmetry breaking, when two-stranded boson 
Elementary (real) bosons are simple configurations of 1, 2 or 3 strands that propagate:

\section{1 strand: photon}

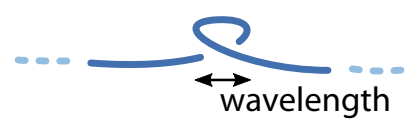

Spin

$\mathrm{S}=1$

2 strands:

$\mathbf{W}_{\mathbf{1}}, \mathbf{W}_{\mathbf{2}}$ (before symmetry breaking)

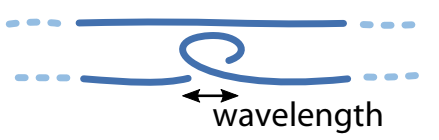

$\mathrm{S}=1$

wavelength

$\mathbf{W}_{\mathbf{3}}$ (before symmetry breaking)

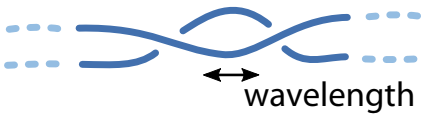

$\mathrm{S}=1$

3 strands:

eight gluons

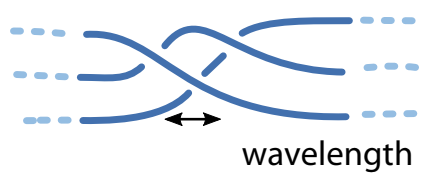

Higgs boson

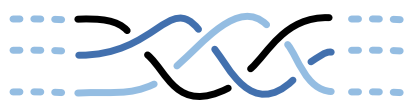

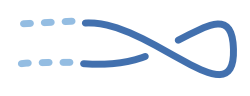

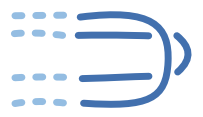

$s=0$

Virtual

bosons:

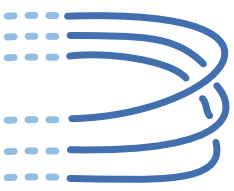

Resulting

observation:

Weak (real) vector bosons after

SU(2) symmetry breaking

(only the simplest family members) with each triple of strands lying flat in a plane:

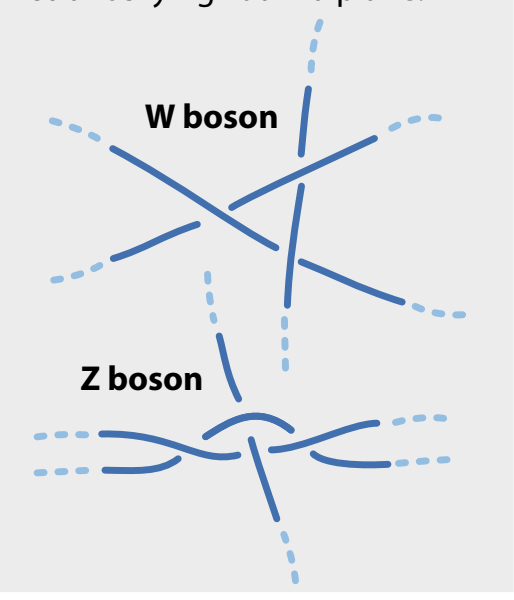

Figure 6: The conjectured rational tangles for every elementary boson. The tangles are made of one, two or three strands. For each boson, the advancing tangle determines the spin value and the propagator. Their spin is 1 , because any curved strand can rotate by $2 \pi$ and return to its original shape. All boson tangle cores rotate when propagating. Photons and gluons are massless, and each are described by just a single tangle. The $\mathrm{W}$ and $\mathrm{Z}$ tangles are asymptotically planar. The $\mathrm{W}, \mathrm{Z}$ and Higgs are localizable and thus have mass; therefore they have additional, more complex tangles in addition to the simplest shown here (see text). No further elementary boson (apart from the graviton [8]) is predicted to exist. The $\mathrm{W}$ tangle is the only topologically chiral one, and thus the only electrically charged elementary boson.

tangles incorporate a vacuum strand, they yield the three-stranded $\mathrm{W}$ and $\mathrm{Z}$ bosons. The complete overview of boson tangles is given in Figure 6. No additional elementary gauge boson appears possible: higher number of strands are not possible in elementary particles, and more complex tangling of the strands of bosons would yield fermion behaviour instead. Photon and gluon tangles are massless, because they can rotate unhindered by tethers, whereas the $\mathrm{W}$ and the $\mathrm{Z}$ boson have mass. 
Quarks - 'tetrahedral' tangles made of two strands (only simplest family members)

Parity $P=+1$, baryon number $B=+1 / 3$, spin $S=1 / 2$

charge $Q=-1 / 3$

d quark

$\mathrm{Q}=+2 / 3$

u quark

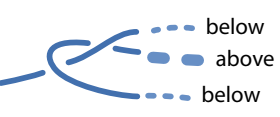

s quark in plane
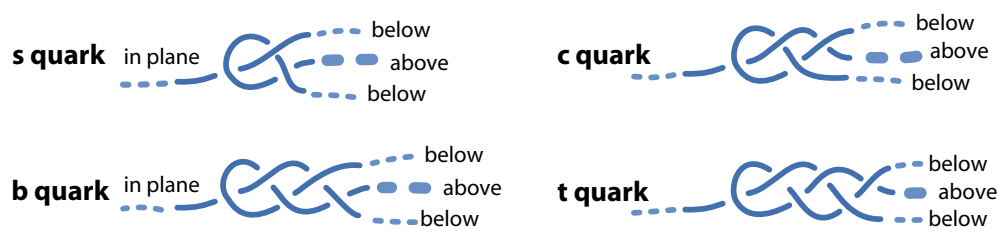

Observation

Leptons - 'cubic' tangles made of three strands (only simplest family members)
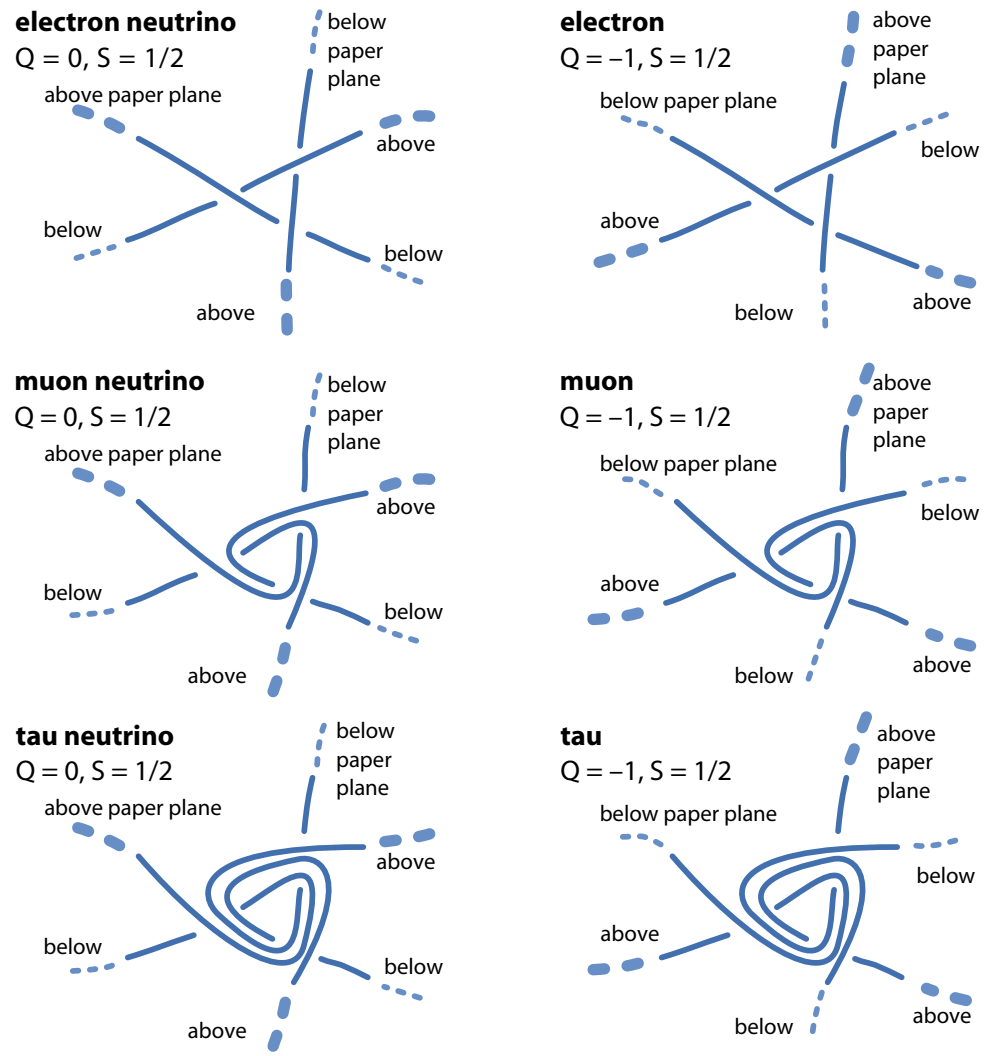

Figure 7: The simplest conjectured tangles for each elementary fermion. All tangles are made of two or three strands. Elementary fermions are rational, i.e., unknotted tangles. The cores are localizable and realize the belt trick. Tangles generate spin $1 / 2$ behaviour, positive mass values, and exactly three generations. The fermion tangle structure leads to Higgs coupling, as illustrated in Figure 12 At large distances from the tangle core, the four tethers of the quarks follow the axes of a tetrahedron. At large distances from the core, the six tethers of the leptons follow the three coordinate axes. Neutrino cores are simpler when seen in three dimensions: they are twisted triples of strands. Note that neutrino cores are chiral but not topologically chiral: thus they are electrically neutral. The tangles of the electron, the muon and the tau are topologically chiral, and thus electrically charged. All massive particles have additional, more complex tangles in addition to the one shown here (see text). No additional elementary fermions appear. 
Test 5: The discovery of additional elementary gauge bosons would falsify the tangle model.

The Higgs boson is a braid made of three strands. For all massive particles, Higgs braids can be added to the tangle core. Every massive particle - fermion or boson - is thus described by an infinite family of tangles that contain a simple core, that core plus one Higgs braid, that core plus two braids, etc. The mass value is influenced by this - single or multiple - Higgs boson addition, as illustrated in Figure 12. That figure also shows that the Higgs couples to itself; it is thus massive. Because no addition of a Higgs braid to cores of massless elementary particles is possible, massless elementary particles are described by a single tangle.

Test 6: The discovery of additional Higgs bosons would falsify the tangle model.

Elementary fermions can consist of two or three strands. One-stranded particle tangles cannot have spin $1 / 2$ nor have mass because the belt trick is not applicable to them. Two-stranded fermions are quarks, three-stranded fermions are leptons. The simplest specific tangles are given in Figure 7. All massive elementary particles have additional tangles: each one are described by an infinite family of tangles that contain the simplest core, that simplest core plus one Higgs braid, the simplest core plus two braids, etc. Strands thus reproduce Yukawa coupling.

Both quarks and leptons are limited to three generations by the coupling to the Higgs, and by the three-dimensionality of space. Figure 8 shows how the infinite class of quark-like braids is split into six infinite families, corresponding to the three generations. The infinitely many possible quark tangles consist of $6+6$ separate infinite tangle families in which each tangle and mirror tangle differs from the other by one or several Higgs braids. Each infinite tangle series corresponds to a quark or to an antiquark.

The quark-tangle assignments in Figure 7 reproduce the quark model of hadrons [7, 28], including the correct retrodiction of which mesons violate CP symmetry; they also reproduce all meson and hadron mass sequences.

The lepton tangle assignments and the quark tangle assignments reproduce the weak interaction. Particle mixing is explained in reference [6]. The neutrino assignments explain their handedness and their small mass. Additional elementary fermions are not possible: they cannot have one strand, nor can the have four or more strands; and if they have two or three strands, they are already included in the infinite families.

Test 7: The discovery of additional elementary fermions would falsify the tangle model.

In summary, in the strand conjecture, tangle classification leads to the fermion and boson spectrum observed in nature. Every observed quantum number is due to a topological property of particle tangles, more precisely, to a topological invariant. (In contrast, the fundamental constants are due to (averaged) geometric properties of tangles.) The appearance of the gauge groups from the boson structure is summarized below.

Test 8: The discovery of any new elementary particle of any kind - such as anyons, axions, supersymmetric partners, or any new elementary dark matter particle - would falsify the tangle model. 


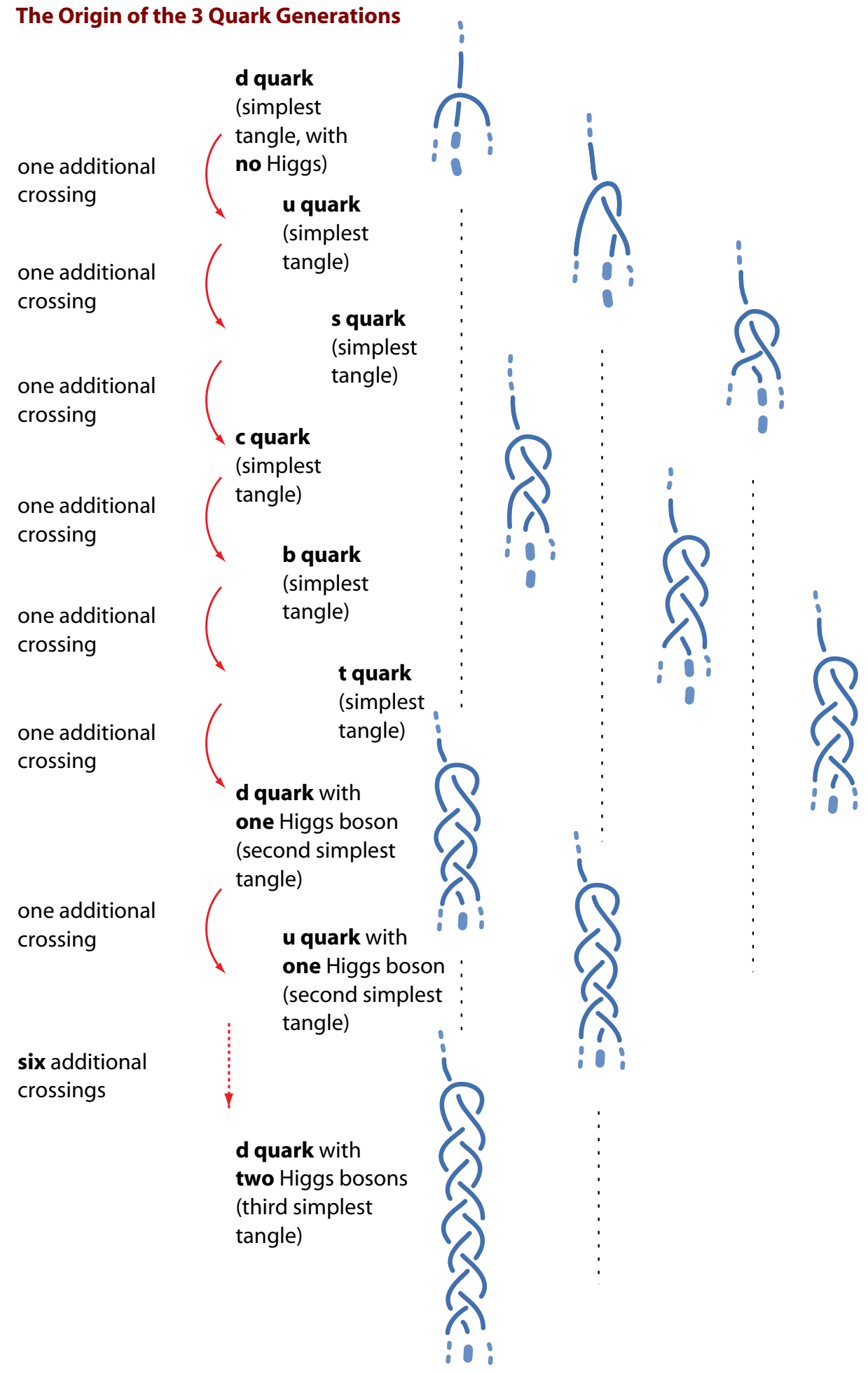

Figure 8: Quarks consist of six two-stranded tangle families, each with an infinite number of tangles. The six families define the three generations. The same happens with anti-quarks, which are represented by mirror tangles. In the strand conjecture, the number of generations is thus due to the Yukawa coupling to the Higgs. 
The strand conjecture for an electron
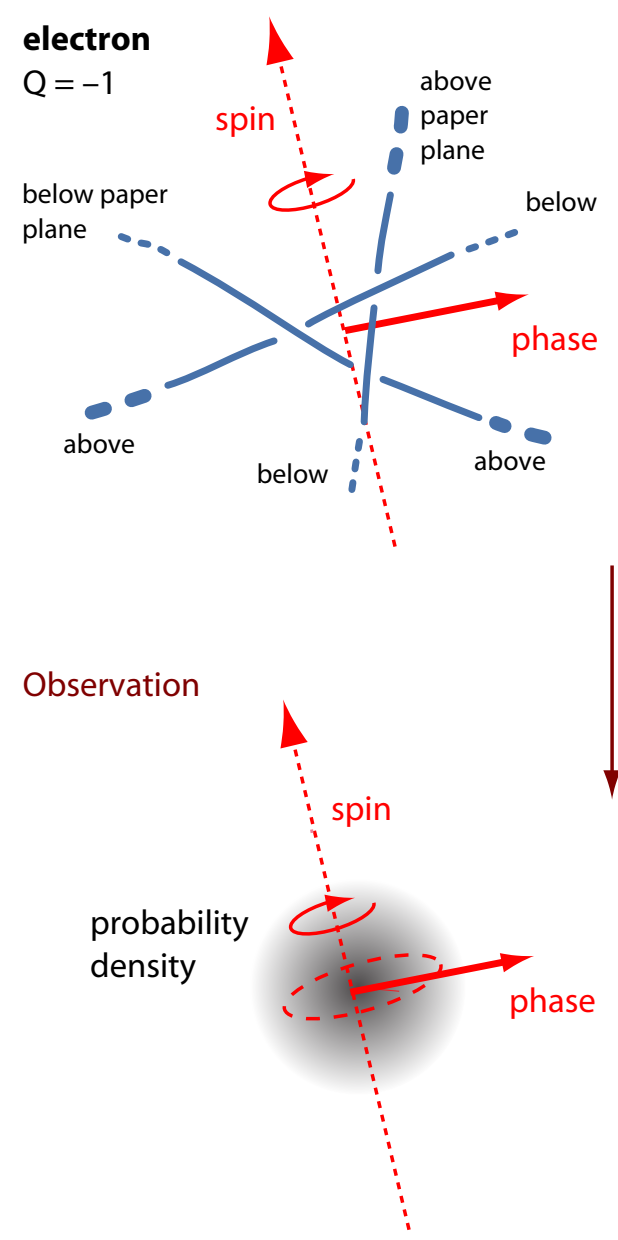

The strand conjecture for a positron

time average of crossing switches

Observation
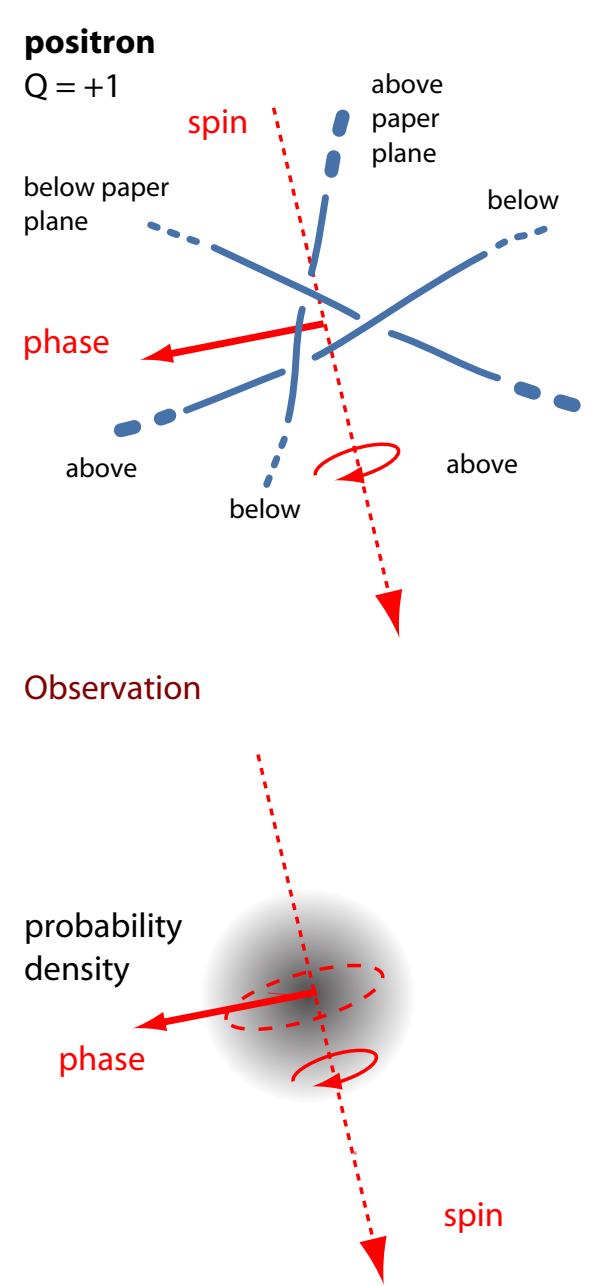

Figure 9: The simplest tangle of the electron (left) can be continuously deformed into the simplest tangle of the positron (right), provided that tethers are allowed to change position.

\section{Predictions about the structure of the electron and other fermions}

In the strand conjecture, elementary fermions are rational tangles [6]. More precisely, elementary fermions are infinite tangle families defined by (1) a simplest rational tangle, plus (2) all those rational tangles that arise when braids are successively inserted at one end. For many fermion properties, it is sufficient to explore the simplest tangle of all the fermion family tangles. The simplest tangles for each fermion are given in Figure 7.

Test 9: Discovering any contradiction between tangle properties and observed particle properties would invalidate the strand conjecture. For example, discovering a new energy scale in high energy physics or any other deviation from the high energy desert would invali- 
date the strand conjecture.

All quantum numbers are due to topological properties and thus are automatically integers or simple fractions. The tangles of the elementary particles determine their helicity and parity, from their mirror behaviour and their tangle core rotation. The tangles determine the magnitude and orientation of their particle spin, from the rotation behaviour of their tangle core. The tangles determine their baryon and lepton number, from the number and spatial structure of tethers. The tangles determine all the other flavour quantum numbers, from the quark content, i.e., from the core topology. Electric charge is explored in detail below, in section 12. The topological origin of strong and weak charge is explored in reference [28].

Test 10: The discovery of forbidden values of quantum numbers, or of the non-conservation of baryon number or lepton number (in processes described by perturbative quantum field theory), would falsify the tangle model.

Test 11: The discovery of new quantum numbers, such as R-parity, would falsify the tangle model.

For the spinning electron, the simplest tangle core is essentially a continuously rotating triangle formed by its three strands. The three crossings each yield a third of the elementary charge. Depending on the spatial approximation, the three rotating crossings can be seen as forming a rotating torus, or, at larger distance, as forming a vortex. At even large distance, the electron is a point particle. In this sense, the tangle structure resembles many other proposed electron models proposed in the past, e.g., by Hestenes [22, 23].

The electron tangle also allows a smooth transition (by rotating certain tethers against the others) to the positron tangle, thus a smooth transition between tangle and mirror tangle. The transition is illustrated in Figure 9. Therefore, the tangle model visualizes the properties of both the electron and the positron. Above all, the tangle model is able to visualize that for a given Dirac spinor $\psi(x)$, the ratio between electron and positron probability density can vary from one position $x$ to another. It might well be that the rational tangle model of the electron is the only model in the literature that realizes a smooth transition between electrons and positrons. This smooth transition is intrinsic to the Dirac equation.

In summary, the tangle model of fermions explains spin, translation, wave functions, antiparticles, quantum numbers, and the particle spectrum. This implies

Test 12: Discovering any substructure in elementary particles that differs from tangles of strands - such as preons, rishons, ribbons, Möbius bands, prequarks, knots, tori, or any other localised or extended substructure - would invalidate the strand conjecture.

It has to be stressed that it is still possible that some fermion tangles are wrongly assigned, but that the strand conjecture as a whole remains correct. In particular, the tangles assigned to the leptons need critical scrutiny. 


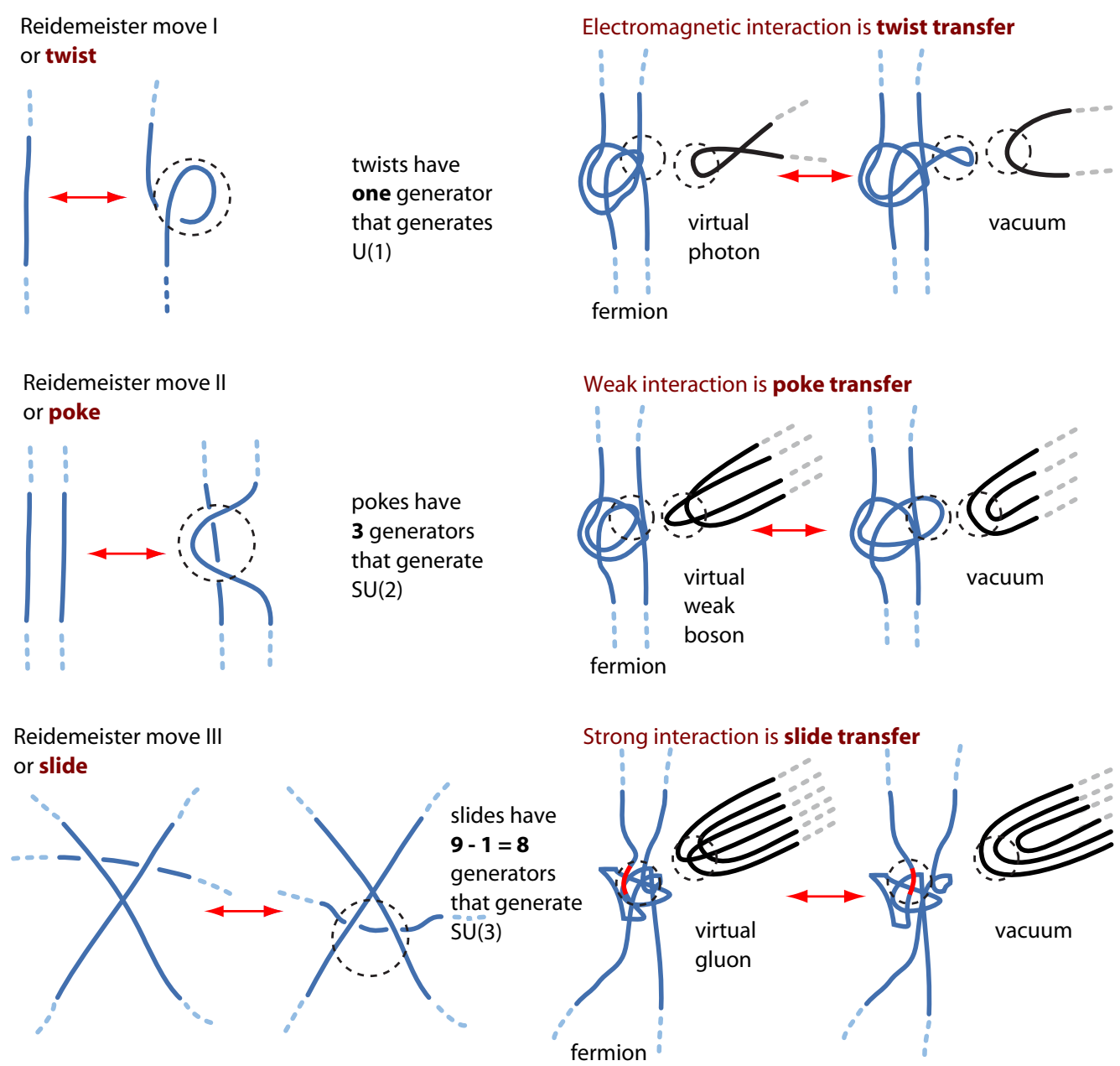

Figure 10: The three Reidemeister moves classify the possible deformations of tangle cores [31]. The moves also determine the generators of the observed gauge interactions, determine the generator algebra, and thus fix the three gauge groups $[6,28]$. Every group generator rotates the region enclosed by a dotted circle by the angle $\pi$. The full gauge group arises by generalizing these local rotations to arbitrary angles. (Taken from reference [7].)

\section{Predictions about gauge interactions}

This section summarizes earlier results [6] showing that interactions are tangle core deformations. This connection is illustrated in Figure 10 Deformations of a localized tangle core modify the phase of the corresponding particle. For example, an externally applied magnetic field modifies the phase of an electron wave function through the absorbed virtual photons.

Deformations of three-dimensional objects are described by gauge groups. In 1926, Reidemeister showed that every tangle core deformation is composed of three basic types: twists, pokes and slides [31]. They are also called the first, second and third Reidemeister moves. The moves have a property that is not widely known [6]: 
$\triangleright$ Tangle core deformations - given by Reidemeister moves - determine the observed gauge groups $\mathrm{U}(1)$, (broken) $\mathrm{SU}(2)$ and $\mathrm{SU}(3)$.

In particular, the gauge group U(1) arises because twists, the first Reidemeister move, can be generalized to arbitrary angles and concatenated. Also, a double twist can be rearranged to yield no twist at all, so that the non-trivial topology of U(1) arises. Electric charge is defined in Section 12 as $1 / 3$ of the (signed) sum of chiral crossings. Electric fields are volume densities of virtual photons, i.e., of twists. Magnetic fields are flow densities of twists. As an automatic consequence, only massive tangles can be electrically charged.

Test 13: Discovering a massless and electrically charged elementary particle would falsify the strand conjecture.

Test 14: Observing the slightest deviation from QED would falsify the strand conjecture.

The gauge group $\mathrm{SU}(2)$ arises because pokes, the second Reidemeister move, can be seen as localized rotations by the angle $\pi$ around the three coordinate axes; they form an SU(2) algebra [6]. The generalization of these rotations to arbitrary angles yield the full SU(2) group. Strands further imply that only massive fermions can exchange weak bosons. Due to the tangle structure of particles, maximal parity violation arises: parity violation occurs because the core rotations due to spin $1 / 2$ interfere with the core deformations due to the group $\mathrm{SU}(2)$ of the weak interactions $[6,28]$. In addition, $\mathrm{SU}(2)$ breaking arises: a vacuum strand is included in the massless bosons, leading to the $\mathrm{W}$ and $\mathrm{Z}$ boson tangles [6, 7].

Test 15: Discovering any deviation from the known (electro-) weak interaction properties of the standard model would falsify the strand conjecture.

The gauge group SU(3) arises because slides, or third Reidemeister moves, reproduce the algebra of the eight generators of SU(3). This is the main result of the previous paper [6]. The full gauge group SU(3) arises because slides can be seen as local rotations by $\pi$, and these rotations can be generalized to arbitrary angles. $\mathrm{CP}$ violation does not and cannot occur in the strand conjecture for the strong interaction. Color charge is given by the orientation of the three-ended side of a quark tangle in space. Color fields are densities of virtual gluons. As a result, usual QCD arises.

Test 16: Observing the slightest deviations from QCD or the usual strong interaction properties would falsify the strand conjecture.

As a note, strands also explain the usual gauge group representation for each elementary particle. Therefore,

Test 17: Discovering any particle with a different representation behaviour would falsify the strand conjecture.

As a second note, strands explain why complex numbers, quaternions and octonions each play a role in one gauge interaction: 
Test 18: Discovering that any other number field describes any observed interaction would falsify the strand conjecture.

As a third note, the visualization of the three gauge interactions agrees with and deepens the ideas of Boudet [32].

Finally, because there are only three Reidemeister moves, strands predict the lack of any other gauge interaction:

Test 19: Discovering a larger gauge group, or a different gauge group, or the corresponding elementary gauge bosons, would falsify the strand conjecture.

In summary, strands imply that there are no interactions beyond the standard model.

\section{Predictions about the standard model}

In the strand conjecture, all Feynman diagrams of the standard model with massive neutrinos and PMNS mixing are recovered. This result from references [6] and [7] is summarized in Figure 11 and Figure 12. Because the lack of other elementary particles, and due to the topology of elementary bosons and fermions (including their infinite family members), no other vertices and no other propagators arise. Therefore, combining

- The particle spectrum deduced from tangles (illustrated in Figure 7 and Figure 6),

- The Dirac equation and the corresponding propagator for each massive particle deduced from tangles (see Section 5),

- The Feynman diagrams due to tangles (see Figure 11 and Figure 12),

- The boson Lagrangians with the corresponding boson propagators (deduced for electromagnetism in Section 12 and for nuclear interactions in reference [6]), and

- The fundamental constants - masses, mixing angles and couplings - deduced from tangles (Section 20, references [6] and [7]),

together, the complete Lagrangian of the standard model arises. The standard model thus appears to result from the fundamental principle of the strand conjecture. In particular, the standard model thus appears to arise directly from the Planck scale.

A second, related way to deduce the Lagrangian of the standard model from strands does not make use of Feynman diagrams:

- Rational tangles determine the fermion spectrum - exactly three generations of quarks and leptons - with the observed particle properties: spin, charges, representations, other quantum numbers, and masses (see Figure 7).

- Rational tangles imply that fermion mixings arise and are described by the usual phases and angles (see [6]). 

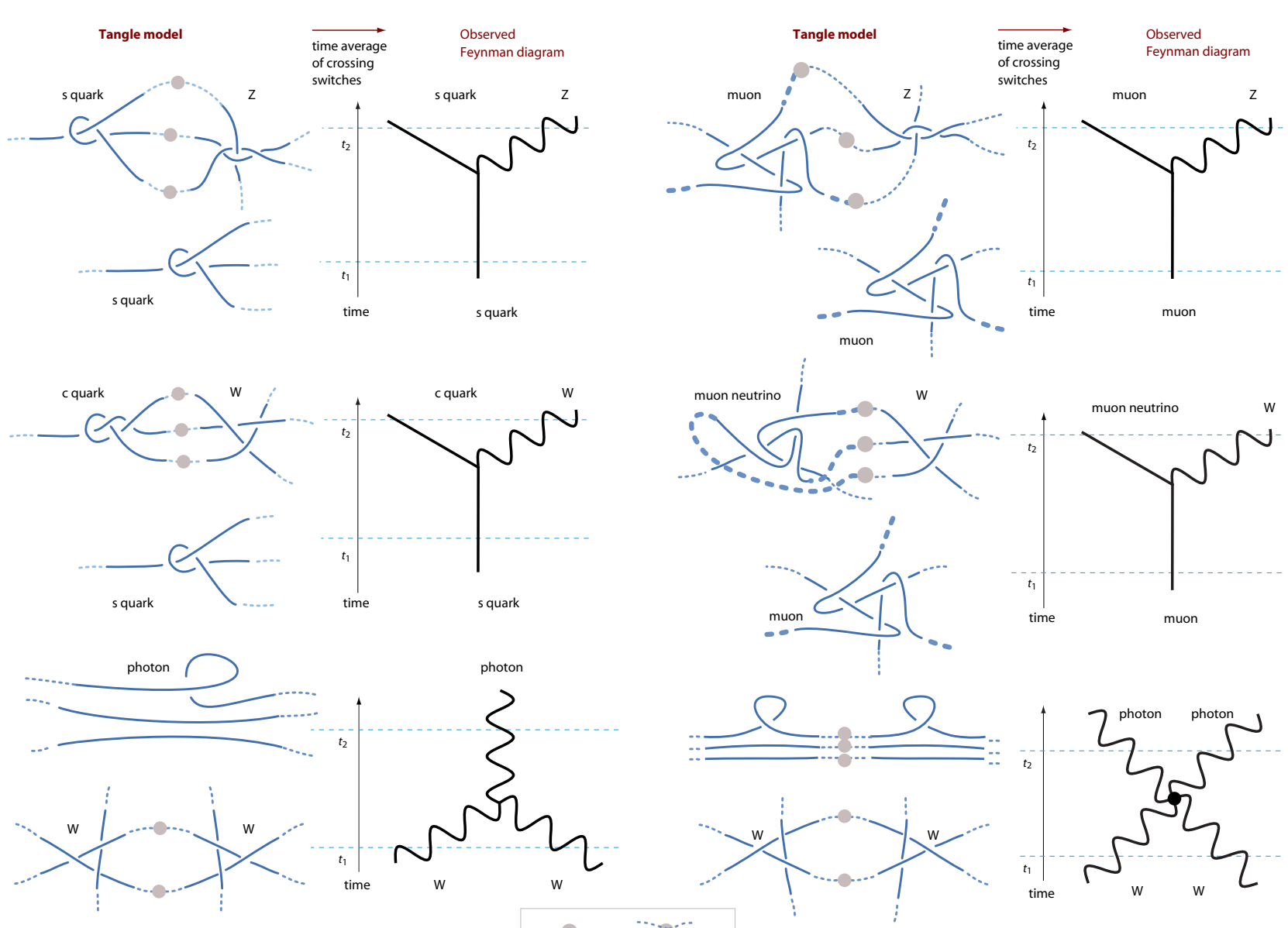

Discs indicate a pair of tethers
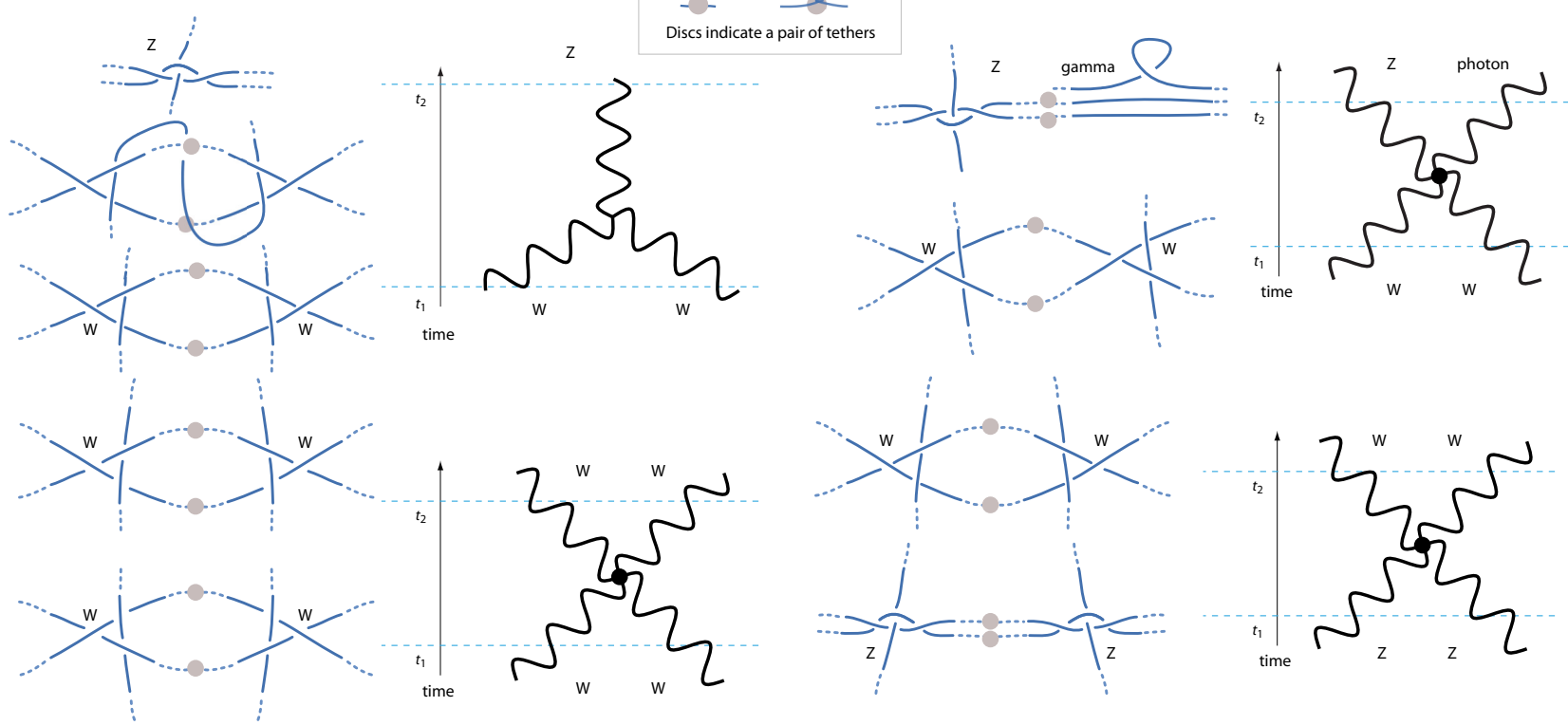

Figure 11: The interaction vertices allowed by fermion and boson topologies imply the complete Lagrangian of the standard model (part one). (Taken from reference [7].) 

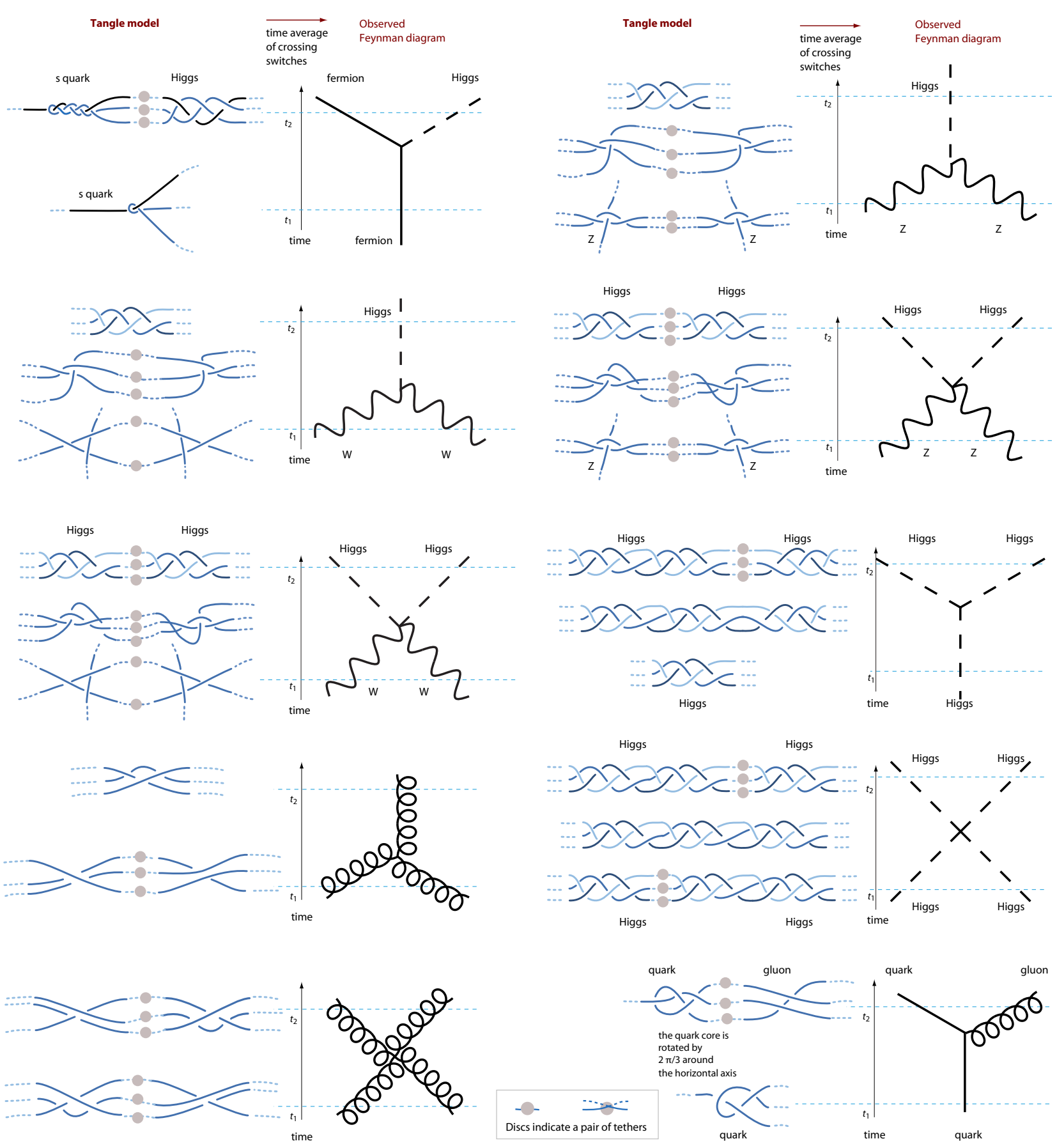

Figure 12: The interaction vertices allowed by fermion and boson topologies imply the complete Lagrangian of the standard model (part two). (Taken from reference [7].) 
- Free fermions tangles are described by free Dirac Lagrangians (see Section 5).

- Tangles determine the boson spectrum with the observed particle properties - spin, charges, representations and masses (see Figure 6).

- Gauge bosons tangles imply that free gauge bosons are described by the usual free field Lagrangians (see Figure 6 and Figure 10).

- Tangle deformations imply that particle interactions are local, simply coupled, renormalizable, have the usual - unbroken or broken - gauge symmetries, obey the conservation of quantum numbers and show unique couplings (see Figure 10).

- The Higgs tangle implies that the Higgs is massive, has spin 0 and is described by its usual Lagrangian (see Figure 6).

- The Higgs boson tangle explains the Yukawa mass terms by braid addition inside tangle families (see also Figure 12).

In total, particle physics appears to be described by the standard model Lagrangian. These results can be summarized:

$\triangleright$ The standard model results from rational tangles.

This connection leaves no freedom of choice; it is clear, definite and unique. The standard model results from tangles because the number of rational tangle families is limited to three generations, and because the number of gauge interactions is limited to three. Above all, the connection can be tested. The tangle model predicts:

Test 20: If any new interaction, such as technicolour or grand unification, any new symmetry, such as supersymmetry, any new elementary particle, or any new interaction vertex that differs from the standard model is observed, the strand conjecture is falsified.

Test 21: If any deviation from the standard model Lagrangian (with massive neutrinos and PMNS mixing) is discovered, at any energy scale, the strand conjecture is falsified.

In summary, the central prediction of the strand conjecture in the domain of high energy physics is:

Test 22: There is no physics beyond the standard model with massive Dirac neutrinos and PMNS mixing. Discovering any new effect beyond the standard model (i.e., any effect apart from the fundamental constants), at any energy scale, would invalidate the strand conjecture.

As a note, the tangle model appears to be free of anomalies. The reasons are the same as those of the standard model [33]. The strand conjecture provides the desired arguments: the lack of anomalies is due to the topology of the particle tangles.

In the following, predictions specific to quantum electrodynamics are deduced. They are all based on the assumption that the standard model derives from strands. If the standard model turns 
The Strand Conjecture for Quantum Electrodynamics

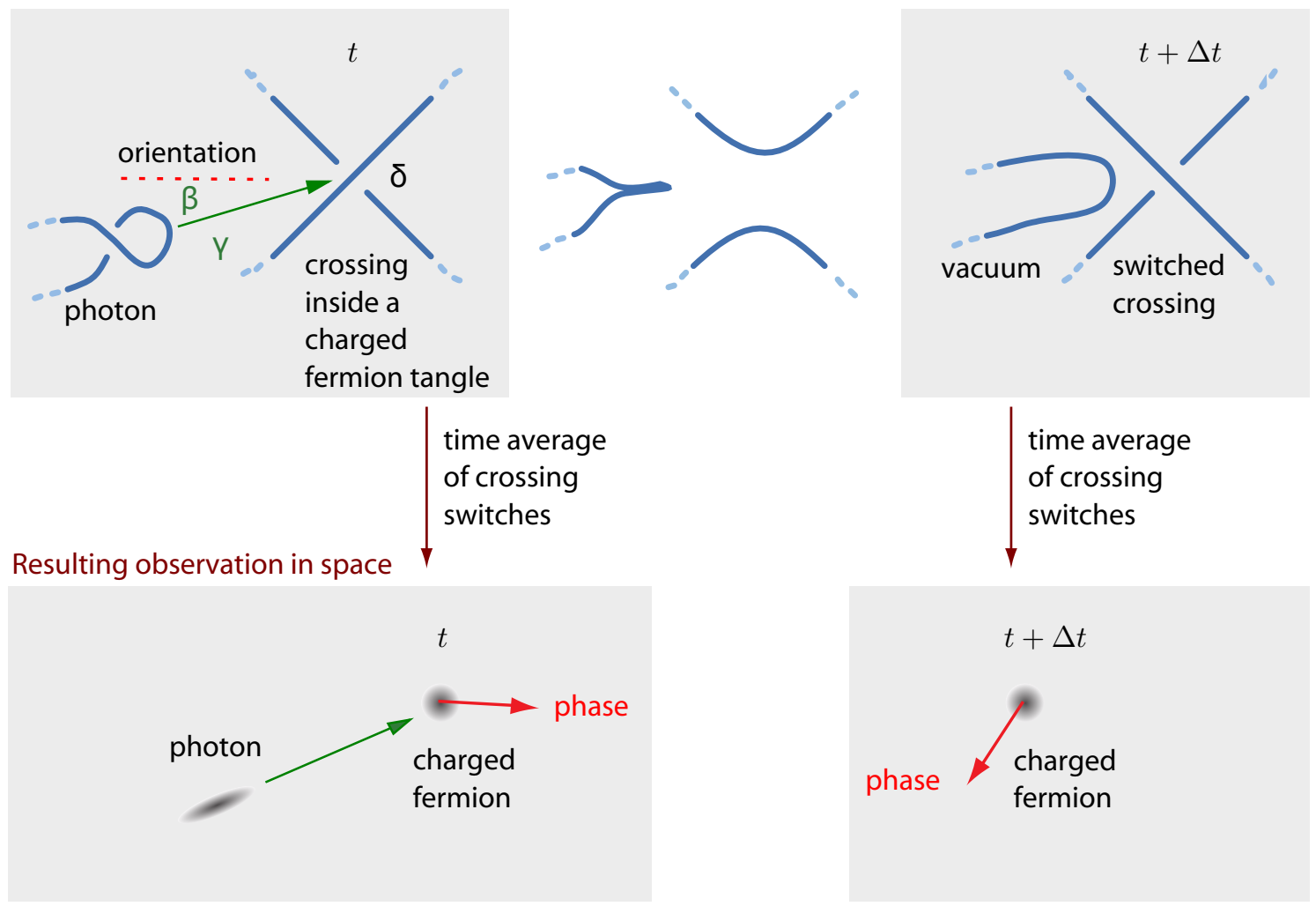

Resulting observation in space-time

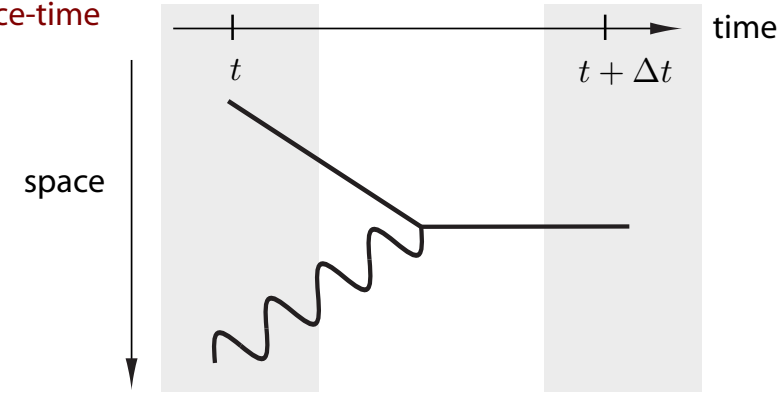

Figure 13: The geometry for the basic process of quantum electrodynamics (QED) is illustrated. Top: the absorption of a photon by a strand crossing, i.e., by a tangle region carrying the charge $e / 3$, at Planck scale. Centre: the corresponding observation at usual scales. Bottom: the corresponding Feynman vertex.

out to be incomplete or even false, the strand conjecture and the following sections are equally false.

\section{The strand description of quantum electrodynamics}

Strands imply that the electromagnetic interaction $[6,7]$ is due to the first Reidemeister move: 
The strand conjecture for QED

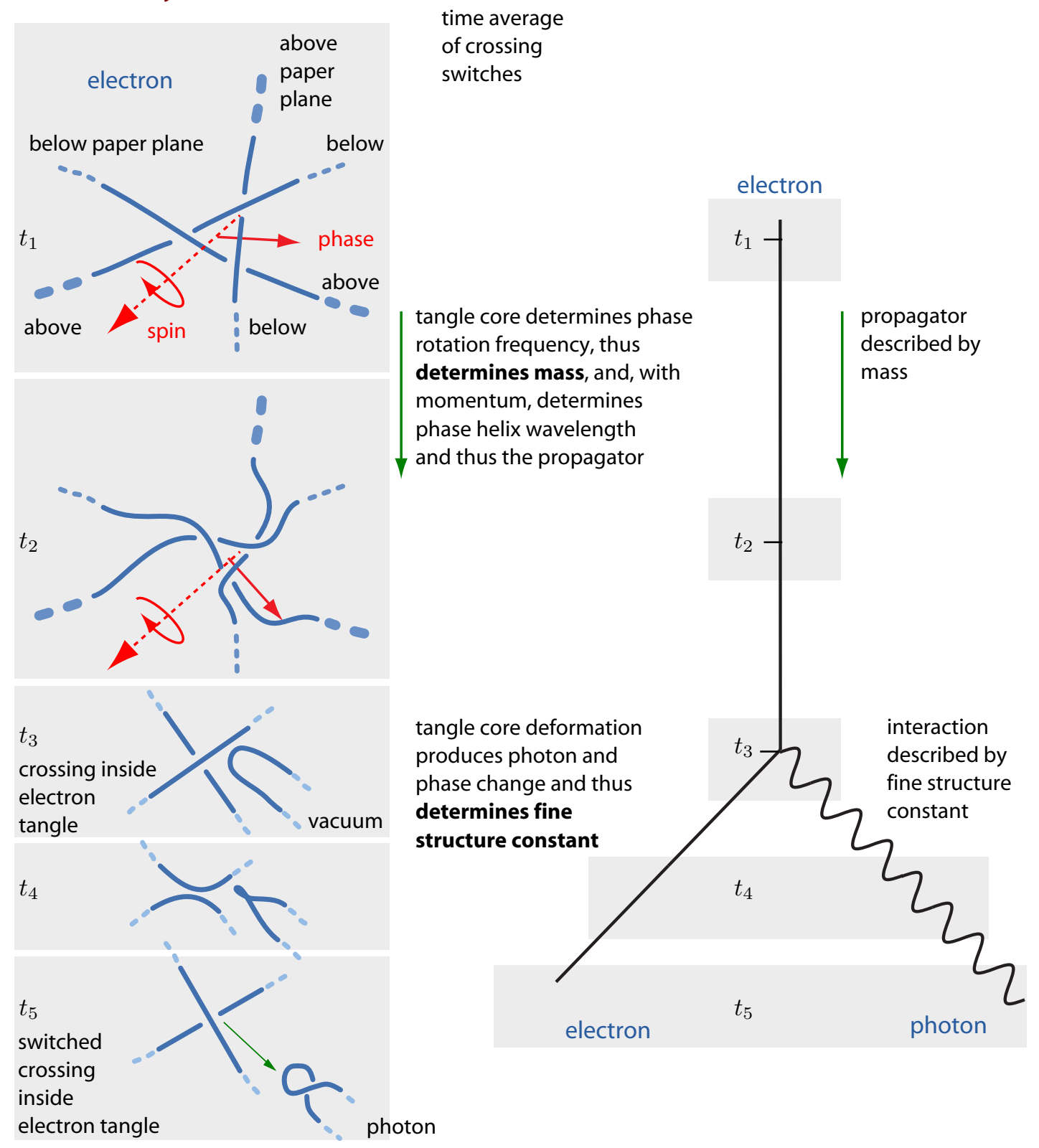

Figure 14: All of QED is illustrated in one picture. In the strand conjecture, the electron mass and the fine structure constant are determined by the electron tangle (here the simplest family member) and its shape change under fluctuations. Like every particle mass, the electron mass is fixed by the average belt trick frequency during propagation. Like every coupling constant, the fine structure constant is determined by the average phase change in the charged particle core (electron in this case) due to emission of a boson (a photon in this case). 
$\triangleright$ The electromagnetic interaction is the - partial or complete - switch of a skew strand crossing in a charged tangle core when a photon twist is absorbed or emitted.

The strand description at the basis of quantum electrodynamics (QED) is illustrated in Figure 13 and in Figure 14. When a photon is absorbed, it transfers its twist to a crossing that is part of a tangle core of a charged particle. The photon (partially) switches the charged tangle crossing and thereby loses its own twist; as a result, it becomes a vacuum strand and the photon effectively disappears. At the same time, the phase of the charged tangle core changes, due to the crossing switch that occurs in the particle tangle. In the corresponding photon emission process, a vacuum strand acquires a twist from a tangle core switch. Again, due to the crossing switch in the particle tangle, the phase of the charged tangle core changes. In other terms, both the absorption and the emission of a photon change the phase of a charged tangle, as is observed. In short, the electromagnetic interaction is due to twist transfers.

In the strand conjecture, a (real) photon is a rotating propagating twist, as illustrated in Figure 6. Photons, being topologically untangled, are massless. Photons have spin 1 because their core is invariant after a rotation by $2 \pi$. Photons, being massless twists, have two helicity states.

Photons advance through vacuum in a way that resembles a localised corkscrew on a strand advancing in a mattress. The mattress is provided by the physical vacuum, i.e., by all other strand segments in the universe. It has to be noted that the photon usually does not advance along its tethers. In addition, the corkscrew can also step over from a strand to a neighbouring one.

In summary, photons have a rotating phase, zero charge(s), vanishing mass, and infinite lifetime.

Test 23: Observing any deviation of the photon propagator from the ideal (strand) photon properties would falsify the strand conjecture.

During the crossing-twist transfer - i.e., the electromagnetic interaction - the phase of the charged particle changes. This connection reproduces the general observation that in nature the phase of wave functions can change in only two ways: either by propagation - as described by the free Dirac equation - or by interaction - as described by the Feynman vertices.

The emission and the absorption of a photon occurs via the removal or addition of a twist in the tangle core. Full twists - rotations by the angle $\pi$ - are illustrated on the top left of Figure 10. Full twists can be generalized to partial twists with arbitrary rotation angles. And partial twists can be concatenated: their angles can be added. In addition, a double full twist can be undone be moving the tethers and is thus equivalent to no twist at all. Together, these properties imply that the concatenation of any two partial twists by the angles $\alpha$ and $\beta$ can be represented by

$$
\mathrm{e}^{i \alpha} \mathrm{e}^{i \beta}=\mathrm{e}^{i(\alpha+\beta)} .
$$

Twists thus define the group $U(1)$.

Because the crossing-twist transfer - i.e., the electromagnetic interaction - arises in a volume of a few cubic Planck lengths, the electromagnetic interaction is effectively local. Because the 
crossing-twist transfer arises in a finite volume of extremely small size, there are no issues with divergences or renormalization. Because strands have a small but finite diameter, a regularization of quantum electrodynamics arises at the Planck scale, and a Landau pole does not arise.

In summary,

Test 24: Observing any deviation from quantum electrodynamics, the U(1) gauge invariance, its locality within Planck dimensions, or the QED Lagrangian would falsify the strand conjecture.

As will be argued now, because any twist transfer is related to topological tangle chirality, the electromagnetic interaction is related to electric charge.

\section{Predictions about electric charge and classical electrodynamics}

In nature, a particle is electrically neutral if its phase does not change when absorbing random photons. In nature, a particle is electrically charged if its phase changes in a preferred direction when absorbing random photons.

In the strand conjecture, the tangle cores of all neutral particles are topologically achiral, i.e., they are equal to their mirror image in the minimal crossing presentation [6, 7]. As a result, neutral particles show no average phase change when they are hit by random photons. In contrast, electrically charged particles have topologically chiral tangle cores. (Topological chirality requires a specific definition for tangles; closure of the two opposite strand ends is implied. With this definition, neutrinos are neutral, whereas electron, muon and tau are charged.) Topologically chiral cores differ from their mirror image in the minimal projection. Topologically chiral cores have a preferred rotation direction when they absorb random photons: they are electrically charged.

It must be stressed that the chirality of tangle structure has no relation to the chirality of interactions.

The strand definition of electric charge implies that it has two possible signs, is quantized, is conserved, and, by definition, is able to emit and absorb virtual photons. Electric charges of particles and antiparticles are predicted to be of exactly the same value, but of opposite sign. Electric charge is predicted to arise only in particles with non-vanishing mass. All this agrees with observation.

Test 25: Observing a massless and electrically charged particle would falsify the strand conjecture.

Electric charge is a consequence of tangle chirality. In all interactions and Feynman diagrams, including those shown below, topological tangle chirality is conserved. As a result, electric charge is conserved. (This also implies that the charge density and the probability density of fermions is conserved.) All this is observed.

Test 26: Discovering an exception to charge conservation would falsify the strand conjecture. 


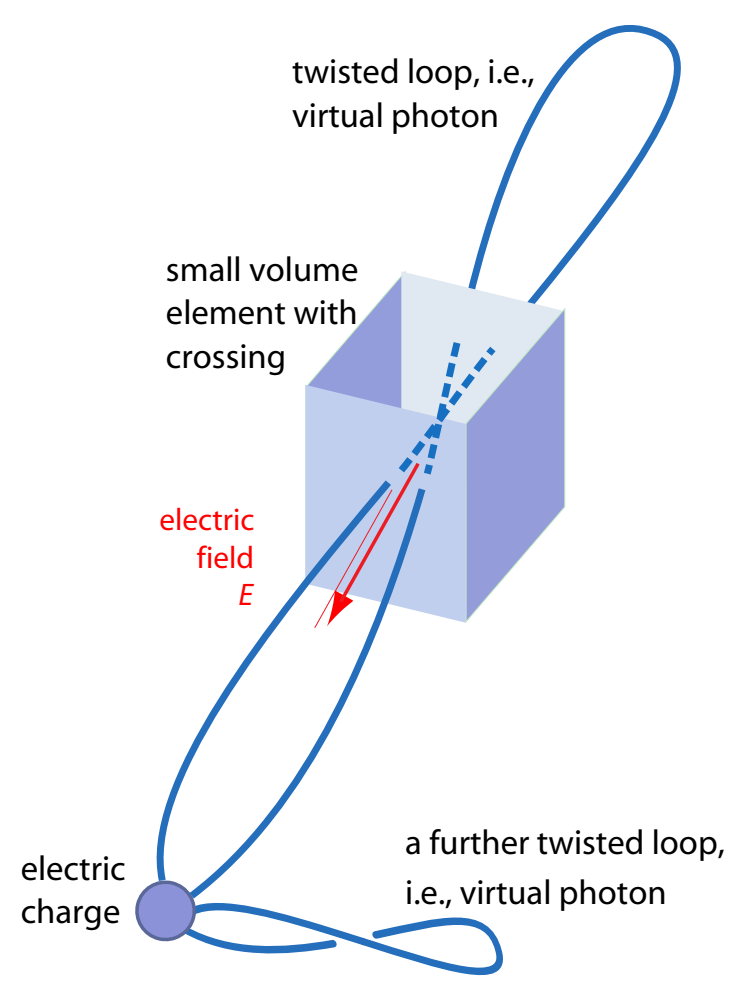

Figure 15: Electric fields - collections of twisted loops, i.e., of virtual photons - arise randomly around a (point) electric charge and lead to Coulomb's $1 / r^{2}$ law. Both for the classical case of a point charge and for the quantum mechanical case - an electric charge due to a tangle forming a probability density - the figure also illustrates minimal coupling. The image, like the model of the photon, also illustrates U(1) symmetry, and the lack of magnetic charges.

All electric charges move more slowly than light, because in the strand conjecture, only massive tangles can be electrically charged. Electrically charged particles thus differ from photons.

$\triangleright$ In the strand description of electrodynamics, the electric field $E$ is the volume density

of twists. The magnetic field $B$ is the twist flow density.

In nature, a static electric charge emits virtual photons. In the strand conjecture, a fluctuating chiral tangle emits twists whose ends are attached to the fermion tangle. Such twists represent virtual photons and are continuously emitted into or transferred to the surrounding vacuum. The electric field - the virtual photons - around a static charge is illustrated in Figure 15. As a result of the vanishing mass of photons and the three dimensions of space, Coulomb's law holds.

Test 27: Observing the slightest deviation from Coulomb's law would falsify the strand conjecture.

Established mathematical arguments now allow deducing an important result. Whenever

- electric charge is conserved, i.e., obeys the continuity equation (in the strand conjecture, this occurs due to the topological definition of charge), 
- all electric charges move strictly slower than light, in Minkowski space-time (in the strand conjecture, this is intrinsic to the tangle model of massive elementary particles), and

- Coulomb's law is valid (automatic in the strand description of electromagnetism as it is due to Reidemeister 1 moves),

then the inevitable consequence, proven by mathematical theorems by Heras and by Burns [34, 35], is:

$\triangleright$ Maxwell's equations hold.

In summary, the strand conjecture implies and predicts classical electrodynamics. The corresponding Lagrangian $L_{\mathrm{ce}}$ of classical electrodynamics,

$$
L_{\mathrm{ce}}=-\frac{1}{4 \mu_{0}} F^{\alpha \beta} F_{\alpha \beta}-A_{\alpha} J^{\alpha},
$$

with $F$ the electromagnetic field tensor, $A$ the four-potential and $J$ the four-current, thus follows from strands. In particular, strands imply minimal coupling. However, one difference to classical electrodynamics does arise.

In the strand conjecture, all physical observables are due to tangle crossing switches. And all strands have a minimum effective diameter. As a result, all physical observables are predicted to have limit values. For electric and magnetic fields, the limits are determined by the maximum force and the smallest electric charge. The maximum local force value $c^{4} / 4 G$ follows from the fundamental principle; however, it already appears in classical general relativity, as shown by de Sabbata and Sivaram, Gibbons, and others $[12,13,14]$. The smallest electric charge in nature is $e / 3$.

Test 28: Observing a field value beyond the corrected Planck limits $E_{\mathrm{cPl}}=3 c^{4} / 4 G e=5.7$. $10^{62} \mathrm{~V} / \mathrm{m}$ or $B_{\mathrm{cPl}}=3 c^{3} / 4 G e=1.9 \cdot 10^{54} \mathrm{~T}$ would falsify the strand conjecture.

The electric and magnetic field limits apply to each component of the electromagnetic field tensor. Up to the present, even the largest observed electromagnetic field values, from particles to magnetars, are several orders of magnitude smaller than these limits. (Similar limits apply to the field values of the nuclear interactions. Also for them, no field values beyond the limits have been observed.)

In the tangle model, electric charge is a topological property of tangle cores, more precisely, a topological invariant. As explained in detail in previous papers [6, 7], every crossing in the minimal projection of a particle tangle leads to an electric charge $+e / 3$ or $-e / 3$. This assignment leads to the observed charge values for all elementary particles. In particular, this assignment thus explains why the charge of the proton is observed to be exactly equal, within measurement precision, to the charge of the positron. This charge equality is not explained in the standard model; in contrast, the tangle model explains it because electric charge is a topological quantity, independent of the particle type. The tangle model seems to be the first explanation for the equality of proton and positron charge in the research literature. 
Test 29: Discovering any exception to electric charge quantization in multiples of $e / 3-$ such as a charge $e / 2, e / 6$ or a millicharged particle - would falsify the strand conjecture.

(A corresponding prediction arises for the quantization of nuclear charges.)

The explanation of electric charge and of its quantization is consistent with an further prediction of the strand conjecture. The tangle model of virtual photons leads to the strand description of electric and magnetic fields. The strand description of electromagnetic fields in turn implies the lack of magnetic charge in nature. Strands do not allow the existence of magnetic charge.

Test 30: The discovery of a magnetic monopole or of a dyon would falsify the strand conjecture.

In summary,

Test 31: Observing any deviation from Maxwell's equations, in the quantum domain or in the classical limit, would falsify the strand conjecture.

\section{Minimal coupling between matter and the electromagnetic field}

Figure 15 illustrates the coupling between charged fermions, i.e., between matter and the electromagnetic field. First of all, the strands in the figure show that the coupling is proportional to the charge: higher charge values have more crossings and thus emit more virtual photons. In an absorption process, higher charge values absorb more virtual photons. The coupling to the electromagnetic field is thus proportional to the charge $q$.

Secondly, the absorbed or emitted photon strand changes the phase of a charge [6]. More photons have a larger effect. This implies that the coupling is proportional to the potential [28].

Thirdly, because the electromagnetic interaction is due to twist exchange, the energy of a charge is changed by the value of the scalar potential times the charge. And because of twist exchange, the momentum of charge - defined by the shape of its probability density - is changed by the vector potential times the charge. These properties define minimal coupling.

Equivalently, strands and twist exchanges visualize and realize the freedom to chose the phase of a tangle; this was illustrated in Figure 4 and Figure 13. The freedom of choosing the phase leads to an U(1) gauge freedom. Strands thus imply U(1) gauge invariance. In particular, strands illustrate that the coupling to the electromagnetic field is equivalent to gauge invariance: both are due to the same geometric effects.

Strands can thus be seen as visualizing the descriptions of Feynman, of Hestenes and of Baylis $[20,21,22,23,36$, 37]: the electromagnetic field is defined by the spacetime rotation rate that it induces on a charge. Strands realize this definition with the help of twist exchange.

In short, strands imply minimal coupling. This property is valid both classically and quantum mechanically. As a result, strands reproduce the full Lagrangian of quantum electrodynamics. In particular, strands reproduce the propagators of photons and of elementary charges, as well as the basic interaction vertex of quantum electrodynamics.

In summary, 
Test 32: Observing any deviation from minimal coupling, at any energy or scale, would falsify the strand conjecture.

\section{Electromagnetism, measurements and minimum time}

Strands explain the electric charge of particles from their tangle topology. Strands also explain the origin of Maxwell's equations. But strands do more: they explain the fundamental principle itself.

The fundamental principle - illustrated in Figure 3 - defines all observations, all measurements and all observables as being due to crossing switches. As discussed above, the basic QED process illustrates that crossing switches are observable precisely because they couple to electromagnetic fields. Every observation process and every measurement device - for measuring length, time, mass or any other physical observable - uses electromagnetic fields. The use of electromagnetic fields is often forgotten - for example when reading the position of a pointer of a weighing scale - but it is essential in every measurement. Without electromagnetism there are no measurements. Every observation, every measurement, and every comparison with a standard are made using electromagnetism. For example, all seven base units of the international system of units (SI) - and thus all other units as well - are defined and realized with electromagnetic means of observation. As another example, all human senses - even hearing - are electromagnetic. Every measurement and every observation is electromagnetic. Strands make this point forcefully, at the most fundamental level. Even though there is no realistic chance to do so, it can be said:

Test 33: Performing any non-electromagnetic observation or measurement would falsify the strand conjecture.

The coupling of crossing switches to electromagnetism also explains the a minimum time $\sqrt{4 \hbar G / c^{5}}$ arises in the fundamental principle. A crossing switch could, in principle, take an arbitrary short time. But such a crossing switch would not and does not couple to the electromagnetic field: a photon wavelength shorter than a (corrected) Planck length is not possible. Such a ultrarapid crossing switch would not be observable; it would not have any physical effect. In short, only crossing switches that take longer than a (corrected) Planck time have physical relevance.

In summary,

Test 34: Discovering any effect whatsoever that is due to time intervals shorter than the minimum time would falsify the strand conjecture.

Test 35: Discovering any effect whatsoever that is due to length intervals shorter than the minimum length would falsify the strand conjecture.

Again, strands predict the lack of any trans-Planckian effects.

\section{Predictions about electric dipole moments}

The tangle model and the photon absorption process illustrated in Figure 13 imply that the charge 'units' $e / 3$ or $-e / 3$ inside an elementary particle are (at high energy) at average distances of the 
order of the Planck length [6]. In particular, the electron tangle contains three charge units of the same sign. The same is true for the charged leptons and the W. In contrast, for the quark tangles, crossing have different signs. In short, strands imply that the intrinsic electric dipole moment $d_{\text {int }}$ is given by

$$
\begin{aligned}
& d_{\text {int }}=0 \text { for elementary particles with charge } e \text { or }-e, \\
& d_{\text {int }} \approx e l_{\mathrm{cPl}} \text { for quarks }
\end{aligned}
$$

These intrinsic dipole values - either zero or negligibly small - are predicted by the tangle model for elementary particles provided that the tangles of Figure 6 and Figure 7 are correct.

In the strand conjecture, additional electric dipole moments arise because charges of opposite sign occur in the perturbation expansion, i.e., through strand fluctuations. These additional electric dipole moments occur in the same way as in the standard model. Strands thus predict the same electric dipole moment values as the standard model, where sizeable electric dipole moments arise only from operators of higher order.

The dipole values predicted by the standard model and those predicted by the tangle model are orders of magnitude smaller than the experimental limits. At present, the tightest experimental limit was for the electron by the ACME collaboration: $d_{e}<1.1 \cdot 10^{-31} e \mathrm{~m}$ [38]. Hopefully, future experiments will allow stricter tests. This is of interest because values for the dipole moment considerably larger than expression (4) are predicted by supersymmetric models.

In summary,

Test 36: Measuring a sizeable electric dipole moment for electrons or other elementary particles would falsify the strand conjecture.

\section{Predictions about the fine structure constant}

In quantum electrodynamics, the fine structure constant can defined in the following way [20, 21, $22,23,36,37]$ :

$\triangleright$ The (average) change of phase induced by the emission or absorption of a photon by a particle of unit electric charge determines the square root of the fine structure constant.

In the strand conjecture, the definition is the same; only the tangle model for particles is added $[6,7]$. The definition can also be generalized to the nuclear coupling constants.

Because the emission or absorption of a photon occurs at a skew strand crossing, the tangle model of QED explains how a charged particle can have a spread-out wave function and nevertheless can behave as (almost) point-like in interactions. On the one hand, the wave function is due to the tangle fluctuations of the complete tangle, which is spread out in space. On the other hand, the electromagnetic interaction occurs at a single crossing, which is effectively point-like. The same applies to the nuclear interactions. 
Because the emission or absorption of a photon occurs via the removal or addition of a skew strand crossing, the value of the fine structure constant is determined by the geometry of the strand process. The same applies to the nuclear interactions.

Because the strand conjecture reproduces all known Feynman diagrams, and because no other gauge groups arise in nature, quantum field theory is predicted to remain valid at all observable energy scales. In particular, all three effective gauge coupling constants run with four-momentum, for the same reason as they do in quantum field theory:

Test 37: Any experimental deviation from the running of the coupling constants calculated with the standard model Lagrangian - including the discovery of a new energy scale or a new symmetry - would falsify the strand conjecture.

Strands imply that all coupling constants, including the fine structure constant, are fixed, unique, calculable and smaller than 1. In particular, the fine structure constant is predicted to be constant over time and space - despite occasional opposite claims. The same is predicted for the nuclear coupling constants. In addition, the strand conjecture predicts that the fine structure constant and the nuclear coupling constants are the same for all particles and for all antiparticles.

Test 38: Any experiment disproving the particle-independence, time-independence or positionindependence of the coupling constants would falsify the strand conjecture.

\section{Using geometry to estimate the fine structure constant}

The switch of a tangle crossing illustrated in Figure 13 allows estimating the fine structure constant $\alpha$ [6]. An improved approximation is presented in the following.

The top of Figure 13 shows the projection along the shortest distance $s$ (defined in Figure 4) of a tangle crossing. In the neighbourhood of the shortest distance, each strand is parallel to the paper plane. Let $\delta$ be the angle between the two strands in this projection. The direction perpendicular to the paper plane is best imagined as the axis of a sphere whose north pole is above the paper and whose south pole is below it. The paper plane then is the equatorial plane. The photon incidence angle $\beta$ shown in Figure 13 is a longitude on this sphere; it can vary from $-\delta / 2$ to $+\delta / 2$. The other photon incidence angle $\gamma$ is the angle from the incident photon direction to the paper plane; it thus corresponds to a latitude and varies from $-\pi / 2$ to $+\pi / 2$.

When a photon approaches a tangle core, it twists the part of the crossing surrounding it. The details of the photon incidence determine the probability $p$ that a crossing switch takes place. The details also determine the value $\nu$ of the induced phase change. Both quantities can be estimated from geometry. The result deduced in the following is more precise than the one deduced previously [6].

The following arguments are based on the geometry of Figure 13 and Figure 16. Because the paper plane is taken to be perpendicular to the shortest distance of a crossing, the orientation axis lies in the paper plane, as illustrated in Figure 16. The phase due to a crossing with angle $\delta$ is best 
described by a vector oriented perpendicularly to the orientation axis, as illustrated in Figure 4 and Figure 16. The freedom of choice of gauge allows taking as phase vector any such vector.

The geometric setting now allows estimating the important quantities. In the first step, the value $\nu$ of the phase change around the orientation axis - that is due to a crossing switch - is estimated. The general contribution of a strand crossing to the total tangle phase is estimated to be $\sin \delta$. A switch that occurs due to a photon incident along the orientation axis of the crossing reverses the crossing phase from the original value to its opposite; the phase change is thus $\nu=$ $2 \sin \delta$. This is the value by which the phase of the total tangle changes when the absorbed photon arrives precisely along the orientation axis. For a general photon incidence, described by the angles $\beta$ and $\gamma$, the induced crossing switch is only partial. The approximate value for the phase change is expected to be

$$
\nu \approx 2 \sin \delta \cos \beta \cos \gamma .
$$

This approximation for the general case completes the first step.

In the second step, the total probability $p$ that a photon induces a crossing switch must be estimated. The probability that a photon induces a crossing switch will vanish for a photon arriving along the poles of the crossing. In other terms, perpendicularly to the paper plane, $p(\gamma= \pm \pi / 2)=$ 0 . In addition, the probability for a crossing switch vanishes for photons arriving perpendicularly to either of the two strands. Furthermore, the switch probability is expected to be highest for the case $\gamma=\beta=0$, i.e., for symmetrical incidence. For such a symmetrical incidence, the switch probability $p$ is easiest to estimate. The probability varies with the crossing angle $\delta$ and with the direction of the induced deformation. Photons can either change the crossing into one of the opposite sign, or change the crossing into a double one, as illustrated in Figure 16. The probabilities for the two processes differ. Decreasing the twist is more probable than increasing the twist, because the available (configuration) volume for a twist decrease is larger than for a twist increase. The difference between the twist-decreasing probability $p_{d}$ and the twist-increasing probability $p_{i}$ yields the total probability value $p=p_{d}-p_{i}$ that is sought. Both probabilities can be approximated.

The first task is the determination of the twist-decreasing probability $p_{d}$. This probability will depend on the complement of the spherical angle spanned by the two strands when they are untwisted. In addition, the probability $p_{d}$ will depend on the ratio between the photon wavelength $\lambda$ and the minimum strand distance $s$ (defined in Figure 4). The effect should yield a pre-factor 1 for $\lambda>s$; the pre-factor should decrease to 0 for smaller wavelengths. After averaging over all values for $\lambda$, in case of symmetric incidence, the twist-decreasing probability will obey $p_{d} \approx$ $(\cos \delta / 2)^{2}$.

The previous reasoning has now to be generalized to a general angle of photon incidence. The approximate twist-decreasing probability $p_{d}$ for a crossing switch is expected to be $p_{d} \approx$ $\cos \theta_{1} \cos \theta_{2}$, where $\theta_{n}$ is the angle between the strand $n$ and the direction of photon incidence.

These approximations yield the probability $p_{d}$ of a twist-decreasing crossing switch for all possible geometries. The angles $\theta_{n}$ are determined by the scalar products $\cos \theta_{1}=(\cos (\delta / 2), \sin (\delta / 2), 0)$. 

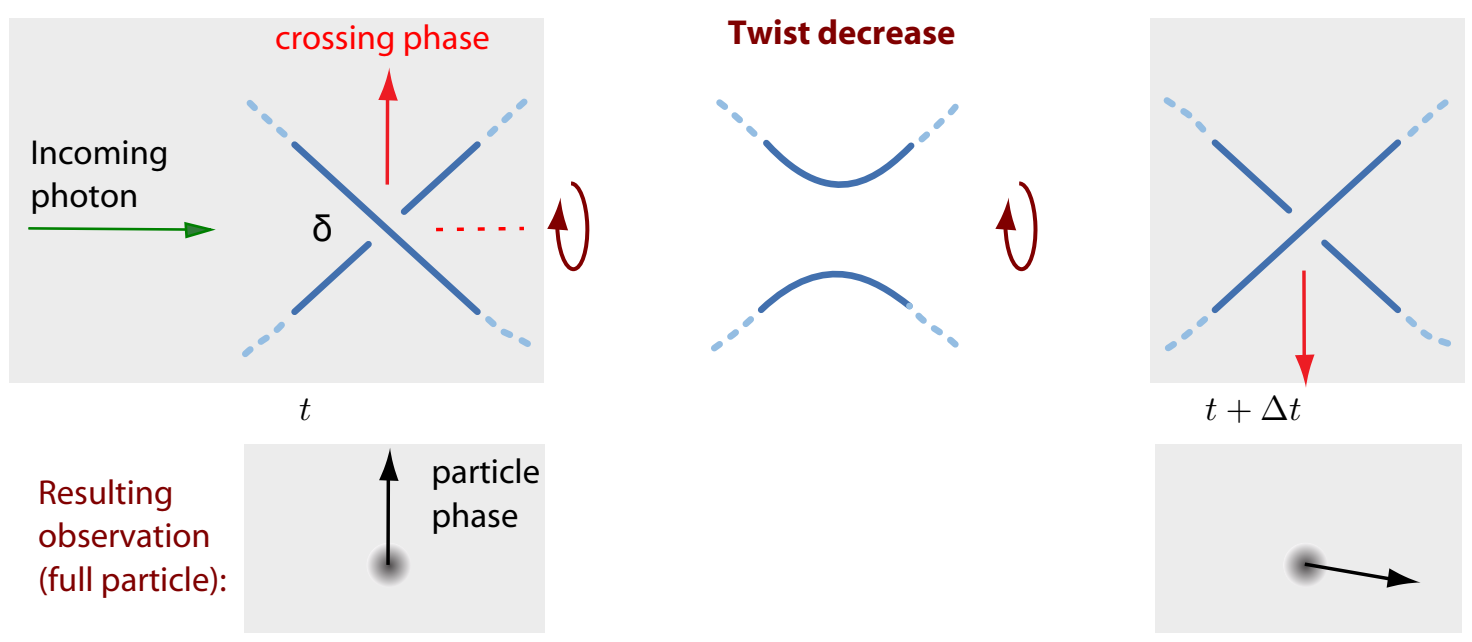

Resulting observation particle
phase
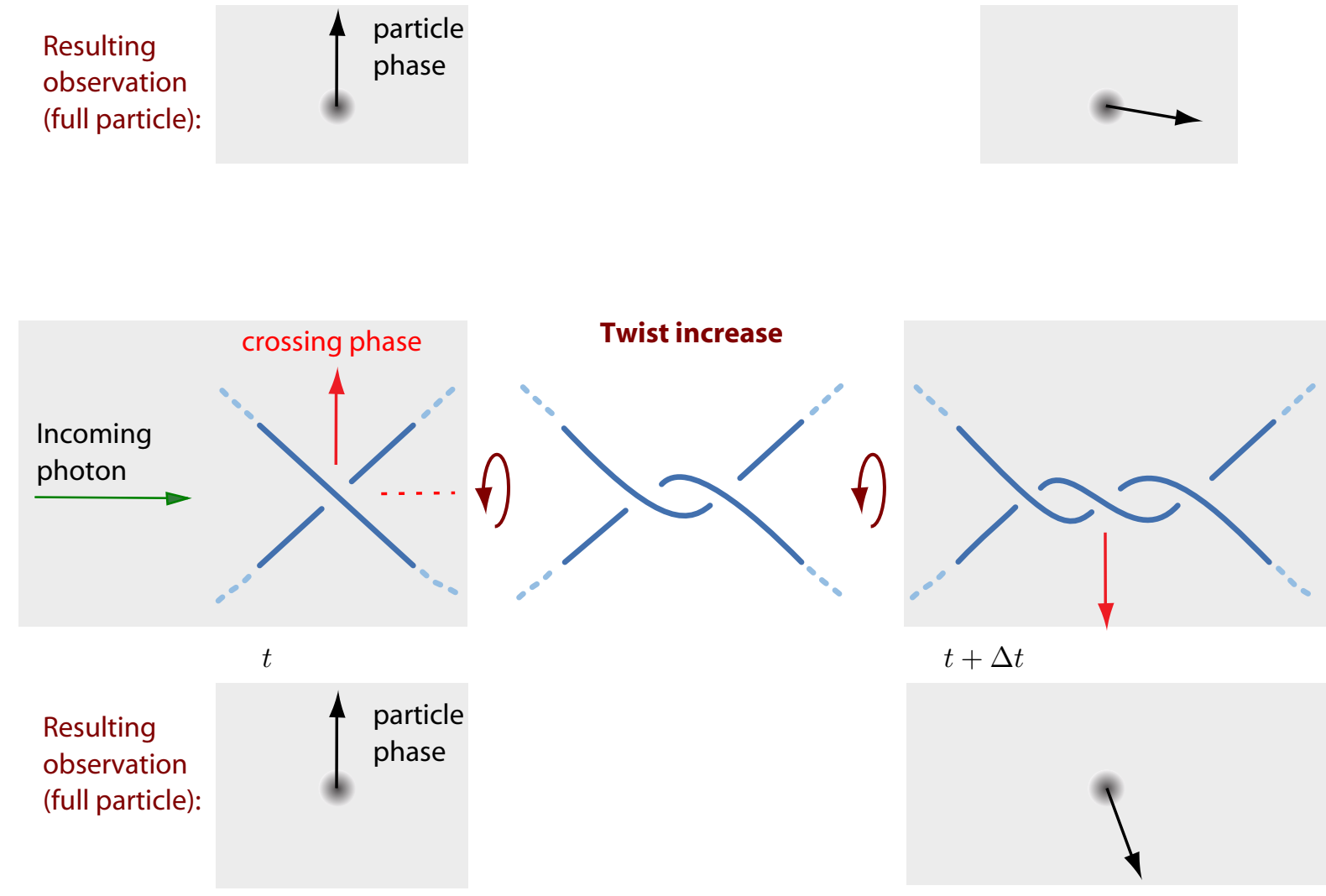

$$
t+\Delta t
$$

Figure 16: Two possible effects of photons (coming from the left) on a crossing are illustrated. Top: the photon can decrease a twist, untwisting it, and continue until the twist changes sign. Bottom: the photon can increase a twist by the same amount. In the strand conjecture, the different probabilities of these two processes appear to determine the value of the fine structure constant (see Section 17). Due to the different probabilities, the effect on the observed phase of a full fermion differs for photons of different helicity.

$(\cos \beta \cos \gamma, \sin \beta \cos \gamma, \sin \gamma)$ and $\cos \theta_{2}=(\cos (\delta / 2),-\sin (\delta / 2), 0) \cdot(\cos \beta \cos \gamma, \sin \beta \cos \gamma, \sin \gamma)$ They yield an approximate probability

$$
p_{d} \approx(\cos (\delta / 2) \cos \beta \cos \gamma)^{2}-(\sin (\delta / 2) \sin \beta \cos \gamma)^{2}
$$


The other task is the determination of the twist-increasing probability $p_{i}$. Like $p_{d}$, also the probability $p_{i}$ will depend on the angle $\delta$ and on the strand distance $s$ (defined in Figure 4). A precise relation between $p_{i}$ and $p_{d}$ can probably only be determined with computer simulations. However, Figure 16 suggests a crude approximation. In the case of a twist increase, the crossing is an obstacle to the approaching photon. The probability for a twist increase might thus be about half the probability of a twist decrease: $p_{i} \approx(0.5 \pm 0.1) p_{d}$. Taking this relation as approximation for all incidence geometries implies

$$
p \approx(0.5 \pm 0.1) p_{d} .
$$

This completes the second step of the calculation.

The third and final step of the calculation of the fine structure constant is the averaging over all possibilities. First of all, the calculation requires averaging the phase change for an absorbed photon times the respective probability. The average has to take place, first of all, over all photon incidence angles $\beta$ and $\gamma$. This requires the use of the spherical surface element $(1 / 4 \pi) \cos \gamma$. Secondly, the calculation requires averaging over all strand crossing configuration angles $\delta$-using the probability density for strand angles given by $\sin \delta$. Thirdly, an average over all photon polarizations is needed; it introduces a factor $1 / 2$. Finally, multiplication by 3 gives the fine structure constant for a full unit charge, i.e., for a tangle core with three crossings. This completes the third and last step.

Combining the three calculation steps, the estimate for the electromagnetic coupling constant becomes

$$
\sqrt{\alpha} \approx \frac{3}{8 \pi} \int_{\delta=0}^{\pi / 2} \int_{\beta=-\pi / 2+\delta / 2}^{\pi / 2-\delta / 2} \int_{\gamma=-\pi / 2}^{\pi / 2} p \nu \sin \delta \cos \gamma \mathrm{d} \gamma \mathrm{d} \beta \mathrm{d} \delta .
$$

Inserting the above approximate expressions for $\nu, p$ and $p_{d}$, gives $\sqrt{\alpha} \approx 0.09 \approx \sqrt{1 / 126}$. This result is, most probably, just a lucky hit. Estimating the total error of the approximations to be $30 \%$ yields

$$
\frac{1}{278} \lesssim \alpha \lesssim \frac{1}{69}
$$

At low energy, the experimental value is 1/137.035999206(11), determined by Guellati-Khélifa and her team [39], and at Planck energy the standard model prediction is 1/110(5). Given the crudeness of the approximations, result (9) still seems too good to be true.

To improve the calculation, first of all, the geometric approximation needs to be improved. It might well be that research on the geometric shape of links, such as the work by Cantarella et al. [40], will be of help. Part of Figure 16 can be seen as depicting what researchers studying geometric link shapes call 'simple clasps'.

Next, the calculation must be corrected for the admixture from the weak interaction. Indeed, in the strand conjecture, the exchange of (half of) the weak $W_{3}$ boson tangle is similar to the exchange of a photon twist. 
Test 39: If a future, more precise calculation of the fine structure constant $\alpha$ based on strands disagrees with measurements, the strand conjecture is falsified.

On the positive side, the Planck scale model for the basic QED diagram remains promising: it reproduces all qualitative aspects of quantum electrodynamics. The approximate value for the fine structure constant is ab initio, unique, constant, and equal for all particles and antiparticles of unit charge.

\section{Predictions about the g-factor and the anomalous magnetic moment}

The exploration of the magnetic dipole moment of elementary particles has fascinated many. A stone near Schwinger's grave shows his formula for the $g$-factor of the electron

$$
\frac{g}{2}=1+\frac{\alpha}{2 \pi}
$$

which is correct to first order in the fine structure constant $\alpha$. The ratio $g / 2$ is defined as

$$
\frac{g}{2}=\frac{\mu / e}{S / m}
$$

with the help of the magnetic (dipole) moment $\mu$, the electric charge $e$, the spin $S$ and the mass $m$. In the past decades, the anomalous magnetic moment $g / 2-1$ of the electron has been measured to a precision better than $10^{-9}$ by Gabrielse's group [41] and calculated, with comparable precision, up to order $\alpha^{5}$ by Kinoshita's group [42].

In the tangle model of elementary particles, spin is due to the rigid rotation of the tangle core. Spin is thus related to the rotation of the mass, whereas the magnetic moment is related to the rotation of the charge. In the strand conjecture, the ratio $g / 2$ can thus be seen as the ratio between two slightly different rotation frequencies, one for mass and one for charge. This conclusion also drawn by Ohanian [43], Sebens [44] and many others - allows deducing a number of tests.

To order $\alpha^{0}$, the tethered core rotates rigidly. In this case, the rotation of the tangle charge is exactly due to the rotation of the tangle mass. Now, the mass value of a particle is due to the motion of the tethers. Due to the belt trick, the motion of the tethers occurs with half the frequency of the core. Thus, $g / 2$ is equal to 1 in the strand conjecture in this approximation. If particles were not tethered, the result would not arise. In short, strands imply that the g-factor, to order $\alpha^{0}$, is equal to 2 for all charged particles. This applies to all those charged systems whose rotating cores make use of the belt trick, independently of the spin value. (The result thus does not apply to a macroscopic charged metal sphere, for example. In this case $g=1$, because the belt trick plays no role in the rotation.) So far, the conclusion agrees with experiment [45] and theory [46, 47]: to order $\alpha^{0}$, all charged fermions, but also the $\mathrm{W}$ boson and all charged black holes, have a g-factor of 2 .

In the tangle model, an expression for the $\mathrm{g}$-factor to order $\alpha^{1}$ arises when in addition to rigid core rotation, also the deformations of one strand segment inside the tangle core are taken into 


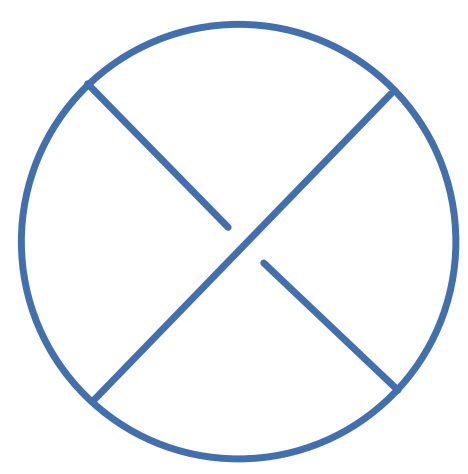

Figure 17: This topological configuration, discussed by Broadhurst and Kreimer, implies that the irrational number $\zeta(3)$ arises in the coefficient of $\alpha^{2}$ for the g-factor. The strand conjecture produces the same configuration, if the circle is seen as representing the tangle core (or its rim), and if the two spokes - the two strand segments being deformed - are seen as the two virtual photon (twists) that leave (and rejoin) the core and point towards the reader. The two photon tangles yield the configuration automatically.

account. In the language of quantum electrodynamics, the expression to order $\alpha^{1}$ arises when one virtual photon is emitted and reabsorbed. In the language of strands, the simplest deformation of the rotating tangle core occurs when one twist, i.e., one virtual photon, is emitted and reabsorbed. This twist emission and reabsorption leads to a phase change of the core. Due to this deformation, when a tangle core rotates due to the belt trick, an effective, additional, small electrical rotation occurs. Like in quantum electrodynamics, the importance of the effect is described by the electromagnetic coupling. Using the definition of the fine structure constant given above, in section 16 , leads to a simple result: To order $\alpha^{1}$, the effect of the core deformation by one virtual photon is an additional phase jump angle given by the fine structure constant $\alpha$. Translated to the rotation situation, this implies that $g / 2$ is larger than 1 by the ratio between $\alpha$ and the full rotation angle $2 \pi$. This is Schwinger's formula (10). An intuitive understanding of Schwinger's formula has been demanded long time ago [48]; strands provide it.

One day it should be possible to determine in a similar way the expression for the g-factor to order $\alpha^{2}$. The expression was first calculated (correctly) by Petermann [49]. The calculation requires to take into account in particular the situation that two virtual photons (i.e., two twists) can also tangle around each other. Interestingly, already some time ago, Broadhurst and Kreimer pointed out a connection between topology and the g-factor [50, 51]. They argued that specific knots imply the appearance of specific zeta values in the expression for the g-factor at higher order in $\alpha$. In particular, they argued that the coefficient $\zeta(3)=\sum 1 / n^{3}$, which appears at order $\alpha^{2}$, is due to the specific topological configuration illustrated in Figure 17. This configuration indeed arises in the strand conjecture when two virtual photons are emitted by an electron tangle.

Following Broadhurst and Kreimer, generalizing the configuration of Figure 17 to more spokes is expected to lead to $\zeta(5)$, which arises at order $\alpha^{3}$, as shown by Laporta and Remiddi [52]. And indeed, such a generalized configuration arises in the strand conjecture when more strand 
segments in the core are deformed, i.e., when more virtual photons play a role. The tangle model for electrons and photons thus might provide an underlying explanation for this research direction and for the relation between topology and the g-factor. The appearance of zeta values might be due to the integrations over the different geometric configurations in space of the twisted loops that correspond to virtual photons.

In summary, the tangle model suggests the following conjecture: the perturbative expression for the g-factor arises due to the effects of photon tangle topology on the multiple integrals that appear in the calculation. This conjecture is supported by the appearance of certain irrational numbers in the coefficients of the perturbative g-factor expansion. If strands agree with the full expression for the g-factor also at higher orders, the tangle model would be confirmed. Whether strands might help in simplifying perturbative calculations cannot be said, at present. But it can be said that the finite number of strands in the finite number of possible leptons leads to a single vertex for quantum electrodynamics, and thus to its renormalizability. As a result,

Test 40: Discovering a contradiction between the tangle model and the g-factor expansion of the standard model would falsify the strand conjecture.

In particular, strands also imply that the standard model calculations will always agree with $g-2$ measurements, despite appearances to the contrary for the muon [53, 54].

\section{Predictions about elementary particle masses}

In the strand conjecture, the fundamental constants are emergent properties. Also the mass of an elementary particle is emergent.

Mass is energy divided by $c^{2}$, and energy is action per time. In the strand conjecture, every crossing switch produces a quantum of action $\hbar$. The (gravitational) mass value of a fermion at rest, in units of the corrected Planck mass, is thus given by the average number of crossing switches that occur per corrected Planck time.

$\triangleright$ The mass value of a fermion is therefore due to the frequency of the spontaneous belt trick that appears due to strand fluctuations [6].

This connection implies a number of predictions about mass that are specific to the strand conjecture and that can be tested even before any mass value is calculated.

Test 41: Because fermion masses are due to the belt trick frequency, particle masses are not quantized - in contrast to gauge charges.

Test 42: Because fermion masses are due to the belt trick frequency, they are surrounded by a cloud of virtual gravitons - which arise in their tethers - that have spin 2, mass zero, and thus lead to a $1 / r^{2}$ dependency of gravity in flat space.

Test 43: In the strand conjecture, only localized particle tangles have mass. In particular, in the strand conjecture, only fermions, W, Z and Higgs bosons interact with the Higgs field, and thus have Yukawa couplings. 
All these predictions agree with observations.

The strand conjecture allows comparing inertial and gravitational mass. On the one hand, the belt trick generates a displacement and thus relates rotation and displacement. In the Dirac equation, this relation is described by inertial mass. Figure 2 gives a (pale) impression of this connection. On the other hand, the double tether twists generated by the belt trick correspond to virtual gravitons; the belt trick thus determines gravitational mass. Because both mass values are due to the same mechanism, in the strand conjecture,

Test 44: Inertial and gravitational mass of particles are intrinsically equal, for all particles, everywhere, at all times.

Strands also predict that

Test 45: The mass values of all elementary particles - due to the respective belt trick frequencies - are positive, fixed, unique and constant in time and space, across the universe.

The mass value of the tangle of an elementary particle is positive, and not vanishing, because the probabilities for spontaneous tangle rotations in two opposite directions differ. The difference is due to the lack of symmetry of tangle cores with respect to rotation: all tangle shapes are helical. (In fact, the simplest tangle for the down quark, shown in Figure 7, is an exception: it is not helical; its other family members are, however. This explains the exceptional order of $u$ and $d$ quark mass values.)

Test 46: Mass values for particles and antiparticles, i.e., for tangles and mirror tangles, are predicted to be equal.

For a system of several particles that are non-interacting, strands imply that the total mass is the sum of the particle masses.

The probability for a belt trick is low. In particular, the probability for the belt trick is much lower than one crossing switch per corrected Planck time. Thus,

Test 47: The strand conjecture predicts that mass values $m$ for elementary particles are much smaller than the (corrected) Planck mass:

$$
m \ll \sqrt{\hbar c^{5} / 4 G}=6.1 \cdot 10^{27} \mathrm{eV} .
$$

The inequality agrees with experiment. It also agrees with the maximon concept introduced long ago by Markov [55]. According to the strand conjecture, the low probability for the belt trick is the main reason that elementary particle masses are much smaller than the Planck mass. The main mass hierarchy is thus explained.

Strands also imply that particle mass values depend on the tangle structure of the particle: more complex tangles - the number of tethers being equal - have larger mass. 
Test 48: In the case of the leptons, strands predict

$$
m_{e}<m_{\mu}<m_{\tau} \quad \text { and } \quad m_{\nu_{e}}<m_{\nu_{\mu}}<m_{\nu_{\tau}} .
$$

The latter prediction, on the normal mass ordering of neutrinos, should be testable in the coming years. The strand conjecture does not allow anomalous neutrino mass ordering. The relation between tangle complexity and mass is valid for all hadrons, as shown elsewhere [6, 7].

In the strand conjecture, particle tangles, and in particular the cores of tangles, get tighter and flatter at higher four-momentum or energy. The flattening will influence the frequency of the belt trick and change the mass value.

Test 49: High particle energy leads to a running of particle mass.

In particular, a flatter tangle core will rotate more easily. Strands thus suggest that lepton mass values should decrease with energy. This is indeed the case in the standard model, as shown, e.g., by Xing et al. [56].

In the strand conjecture, the running should be calculable with computer simulations.

Test 50: Any deviation from the mass running calculated with strands from the mass running found in experiments would falsify the strand conjecture.

There is even a certain chance that the mass running calculated with the standard model might deviate from the mass running due to the tangle model. In this case, high-energy experiments could test the strand conjecture directly.

\section{Using geometry to estimate the mass of the electron and the other leptons}

In the strand conjecture, the mass value of a fermion is determined by the frequency of the spontaneous belt trick that appears due to strand fluctuations. Because localized tangles are helical, i.e., asymmetrical, the spontaneous belt trick leads to a certain average number of crossing switches per time. This yields an average action value per time, which defines an energy and thus a mass value [7]. The frequency of the spontaneous belt trick, and thus particle mass, can be estimated. At present, this requires approximations.

In the strand conjecture, every massive particle is represented by a family of tangles. As mentioned in Section 7, the family members differ by the number of Higgs braids they contain. In the following, the calculation only takes into account the tangle of the simplest family member. The effect of the other family members - due to higher order Higgs couplings - is neglected in the following.

The second approximation is the assumption that tight tangles are representative for average tangles. Recent research suggests that this is a very good approximation [57]. In the strand conjecture, tangles can be tight or loose. The belt trick occurs in all cases. For extremely loose tangles, the frequency of the belt trick is expected to be independent of the tangle size. However, for tight tangles, the rope or cable diameter will be felt. 


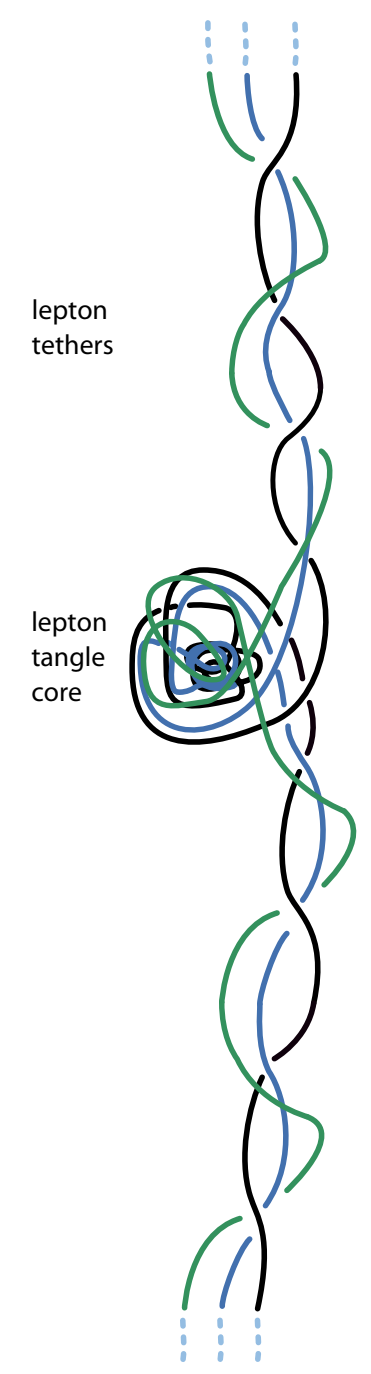

Figure 18: This drawing schematically illustrates one of the most improbable strand shape configurations during the belt trick of a lepton, thus of a tangle made of three strands with its six tethers. The configuration arises after rotation of the tangle core by an angle $4 \pi$ when three tails are put on each end. The geometric shape is important for determining the mass of the electron and the other leptons (see text).

A third approximation is almost automatic. Taking tight tangles as representative ignores the running of mass with four-momentum. For high four-momentum values, the shape of the tangle core will change, and so will its mass value.

On the other hand, using tight tangles to determine mass values implies that the diameter of strands is not neglected - as it was up to this section. In other words, gravity is not neglected in the following. This is as expected: determining particle mass indeed requires taking into account both quantum effects and gravitational effects. 
Estimating the probability for the appearance of the belt trick remains a difficult problem. The research literature does not contain any hint towards a solution. Researchers on polymers, on fluid vortices, on cosmic strings, on string theory, on superfluids, and on statistical knot theory have not explored the topic yet. The following ideas should thus be seen as first tentative steps.

Particle mass $m$ is given by the number of crossing switches per time that occur around the particle. For a fermion made of two strands, the crossing switches are generated by the tethered rotation of the particle that is illustrated in Figure 2. For leptons, which consist of three strands, the configuration after a double core rotation is illustrated in Figure 18, The illustration yields

$$
m \approx p \cdot f \cdot n
$$

In this expression, the factor $p$ describes the probability for the initial double rotation, i.e., the probability to get from the first to the second configuration in Figure 2. For a symmetric core, the probability of a double rotation, whatever the orientation of the rotation axis, is expected to be equal in clockwise and anticlockwise direction. Therefore, for symmetric tangle cores, $p$ is extremely small. For slightly asymmetric tangle cores, as is the case for all rational tangles, the factor $p$ is still expected to be quite small. The value depends on the (averaged, three-dimensional, geometric) asymmetry of the tangle core. The geometric asymmetry is also the quantity that couples to the Higgs braid. A non-vanishing asymmetry leads to a non-zero mass.

In addition, a chiral tangle core, i.e., a charged elementary particle, will have a higher probability $p$, and thus a higher mass, than a neutral tangle core of the same complexity. This explains why charged leptons are more massive than uncharged ones.

In expression (14), the factor $f$ is the belt trick frequency that leads from the second configuration in Figure 2 to the sixth configuration. The factor $f$ will also be small, as the belt trick competes with the inverse double rotation of the tangle core. Interestingly, the small frequency $f$ is expected to be roughly scale independent: the size of the tangle core does not play an important role.

Finally, in expression (14), the number $n$ is the average number of crossing switches per belt trick. The number $n$ counts the crossing switches among tethers and also the crossing switches between the tangle core and the tethers. This number will definitely depend on the size of the tangle core; $n$ increases with tangle core size and tangle core complexity.

The proposed explanation of expression (14) for particle mass $m$ can be checked even before performing any calculation or estimate. Indeed, the explanation yields a particle mass value that is equal for particle and antiparticles, constant over space and time, and not quantized in multiples of some basic number. The explanation yields equal gravitational and inertial mass values. The explanation implies mass values that run with four-momentum, i.e. with the looseness and flatness of the tangle core. The explanation implies mass values which depend, via $p$, on the Yukawa coupling to the Higgs boson. The explanation yields, due to the factor $f$, mass values that are much smaller that the Planck mass. Finally, as expected, due to the factors $p$ and $n$, particle mass values increase for more complex tangles; indeed, large tangles are also more asymmetric. 
At present, a direct calculation of particle mass $m$ with equation (14), even approximate, remains elusive. The main reason is the complexity of Figure 18. However, some rough numerical statements can be made.

Test 51: Strands allow deducing a lower limit for the (bare) mass values of elementary leptons. For the electron neutrino, the probability $p$ of rotation results from the averaged asymmetry of its tangle core. For an electron neutrino, the asymmetry is extremely small, and results from the mixing with the other two neutrinos or from the Yukawa term, or from both effects together. The averaged asymmetry is expected to be of the order of parts per million:

$$
p \approx 10^{-6}
$$

An error of a few orders of magnitude is expected.

The belt trick frequency $f$ for a neutrino results from the probability that the belt trick occurs despite competition with the backwards rotation of the core. To occur, a tether configuration has to form six circles around the tangle core with the same orientation, one for each tether. The size of the six circles is not important. For each tail, the probability is roughly given by the probability to form a circle divided by the number of possible rotation axes and the ways to distribute the tethers along the two ends of each axis. This yields

$$
f \approx\left(\frac{\mathrm{e}^{-2 \pi}}{6 \cdot 4 \cdot 2}\right)^{6} \approx 3 \cdot 10^{-27} .
$$

where six tethers determine the exponent. The systematic error is expected to be several orders of magnitude.

With their 6 tethers, the number $n$ in the mass expression (14) for leptons is surely larger than 24 , which is found by counting the crossing switches among the tethers alone.

The lower mass bound $m_{11}$ for leptons thus is

$$
\frac{m_{11}}{\sqrt{\hbar c^{5} / 4 G}}=p \cdot f \cdot n \approx 10^{-31}
$$

i.e., of the order of $\mathrm{meV} / \mathrm{c}^{2}$. So far, this lower limit is not in contrast with the present experimental limits on neutrino mass, which the KATRIN experiment set at $0.9 \mathrm{eV}$ [58]. However, the difficulty of deriving a reliable lower mass bound is evident. If a future, improved calculation of neutrino masses is in contrast with future data, the tangle model is falsified.

Test 52: For the electron, with its chiral tangle core shown in Figure 7, the averaged asymmetry $p$ of its tangle is expected to be of the order $\mathrm{O}(1)$. This yields

$$
\frac{m_{\text {electron }}}{\sqrt{\hbar c^{5} / 4 G}} \gtrsim 10^{-25}, \text { or } m_{\text {electron }} \gtrsim 1 \mathrm{keV} / c^{2} \text {. }
$$


The limit is not in contrast with the measured electron mass of $511 \mathrm{keV} / c^{2}$. If a future, improved calculation is in contrast with experiment, the tangle model is falsified.

Test 53: Strands also allow deducing an upper limit for the mass values of the leptons. For the most massive lepton, the estimate $n \approx 24$ used for the lower mass limit will remain valid. The estimate for $f$ will change for tangle cores that are elongated; the factor $(6 \cdot 4 \cdot 2)^{6}$ will be of the order of $O(10)$. Again, the probability $p$ due to the asymmetry of the charged leptons will be of the order $O(1)$.

As a result, the upper mass limit for leptons is given by

$$
m_{\mathrm{ul}} \approx 10^{15} \cdot m_{\mathrm{ll}}
$$

or around $1 \mathrm{TeV} / c^{2}$. The observed mass for the most massive lepton, the tau lepton, is $1.7 \mathrm{GeV} / c^{2}$. This mass value is several orders of magnitude lower than the upper limit. If a future, improved calculation disagrees with data, the tangle model is falsified. There is an urgent need for better approximations for lepton masses.

Test 54: For the electron mass, an improved estimate of $f$ leads to a improved upper mass limit. In the case of the electron tangle, the factor $(6 \cdot 4 \cdot 2)^{6}$ will be around $6^{6}$. This is the estimate for the number of possible ways to perform the belt trick in the case of the electron tangle. This yields

$$
\frac{m_{\text {electron }}}{\sqrt{\hbar c^{5} / 4 G}} \lesssim 10^{-19}, \text { or } m_{\text {electron }} \lesssim 3 \mathrm{GeV} / c^{2} .
$$

Also this upper mass limit is not in contrast with the measured electron mass value of $511 \mathrm{keV} / c^{2}$. If a future, improved calculation ever disagrees with experiment, the tangle model is falsified.

Test 55: Another example of the connection between mass value and tangle core structure is worth mentioning. Figure 6 and Figure 7 imply that the tangle core of the Higgs boson closely resembles the core of top quark. Locally, both cores are braids with six crossings. Both cores are of high complexity and are not spherical. In fact, their tight core shapes are the most elongated among all elementary particles. For these two elementary particles, the tangle model therefore predicts the highest mass values, and predicts that they are of the same order of magnitude. This is indeed observed.

\section{Discussion}

The upper and lower electron mass limits deduced above are disappointing. The mass estimates have large error bars, essentially because the problem is a composition of several difficult mathematical challenges. The specific tangle topology was not taken into account yet. The running with four-momentum and the effects of the other tangles in the electron tangle family (i.e., the effect of coupling to the Higgs) were neglected. As a result, the upper mass limit is not precise and differs 

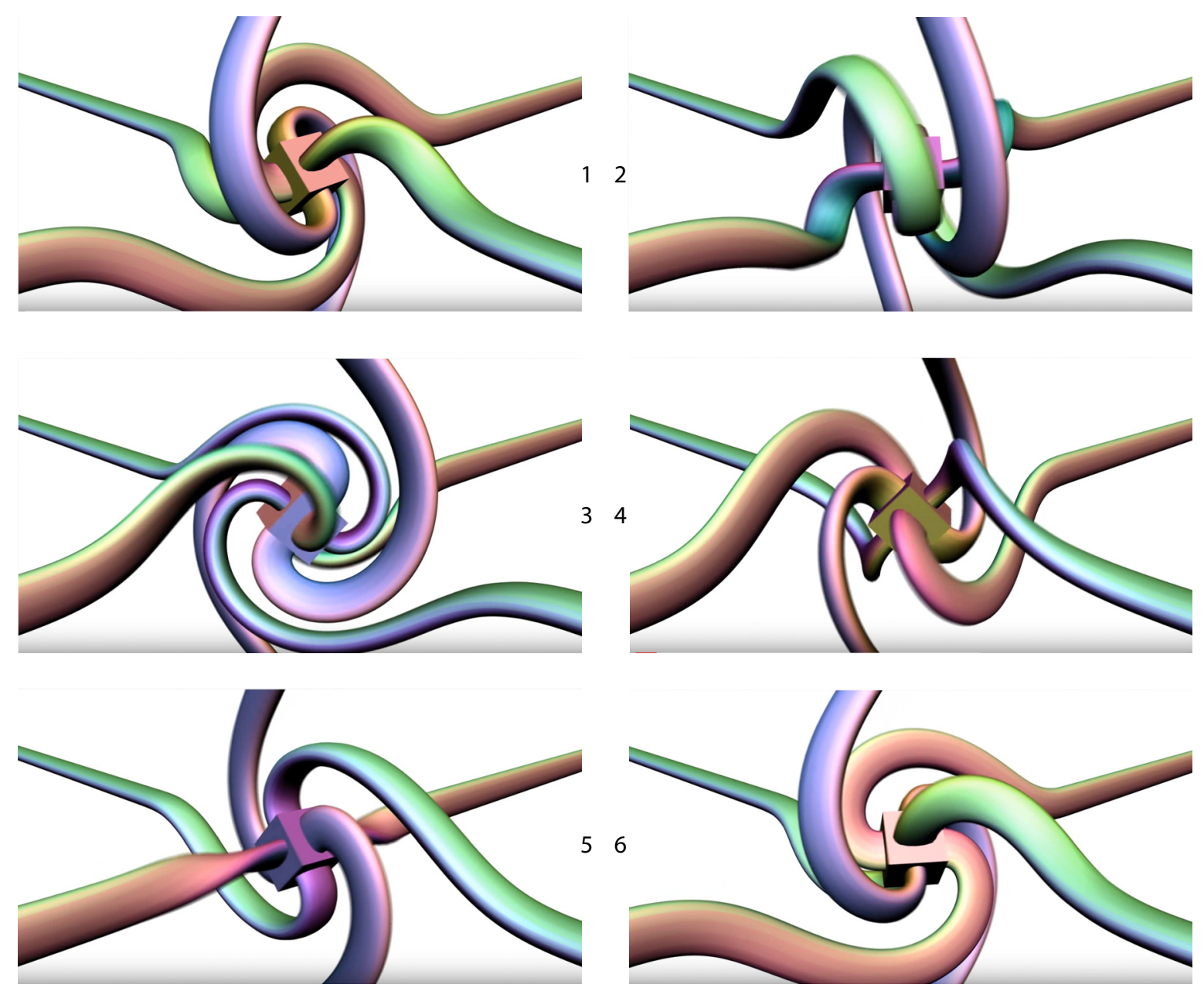

Figure 19: A simple way to visualize particle rotation for a lepton is the animation by Jason Hise

[59]. The rotating central cube symbolizes the tangle core. (Taken from reference [7].)

from the experimental electron mass by many orders of magnitude. Also the lower mass limit is so vague that it cannot be compared to experiments yet.

Despite the disappointing mass limits, two encouraging aspects remain. First of all, the tangle model promises to calculate mass values ab initio. The mass values are unique, constant over time and space, positive, equal for particles and antiparticles, equal to the gravitational mass and running with four-momentum. The mass hierarchy between neutrinos, the charged leptons and the Planck mass is explained - without additional assumptions. More precise estimates of lepton masses, in particular of the electron mass, appear possible either using efficient computer simulation programs or using improved analytical approximations that take tangle topology into account. For example, other shape configurations than the one shown in Figure 18 are candidates for the most improbable intermediate shape. An example can be extracted from Figure 19, It might be that a shape configuration similar to that of subfigure 3 allows a more precise mass estimate for 
leptons. As another example, it might be that work on self-avoiding curves, by Crane and his team [60], will be of help in this exploration.

Test 56: The failure to reproduce, with more precise calculation methods, any one of the observed lepton, quark, W, Z or Higgs mass values, at any single four-momentum value, would falsify the tangle model.

The same test arises also for the various mixing angles and CP phases, though these quantities were not discussed in this work.

The other encouraging aspect of the strand conjecture is the potential to determine, using the same model, the fine structure constant, again ab initio. The predicted value for the fine structure constant is unique, is constant over time and space, runs with four-momentum, and is the same for all particles. These predictions are not made by any other model. Because there are fewer approximations in the case of the fine structure constant, the goal of a precise determination appears closer than for the electron mass.

Test 57: The failure to reproduce, with more precise calculation methods, the observed value of the fine structure constant $\alpha$ or of the nuclear coupling constants would falsify the tangle model.

\section{Conclusion}

The tangle model for elementary particles follows from the strand conjecture. Propagating matter particles are modelled as advancing rotating tangle cores. The electromagnetic interaction is modelled as twist exchange between a photon tangle and a charged fermion tangle.

The tangle model describes nature with a single and simple principle: All observations are due to crossing switches at the Planck scale of fluctuating, unobservable strands. The tangle model implies that all dynamics in nature, and in particular the complete Lagrangian of the standard model, can be described with a mixture of topology and three-dimensional geometry. Despite these unusual aspects, the strand conjecture agrees with all experiments so far.

The tangle model deduces that the standard model is consistent and valid at all experimentally accessible energies. The tangle model predicts that high energies will yield no new, unknown phenomena. The model predicts that the only new aspect of high energy physics to be discovered is the origin of the fundamental constants.

The tangle model cannot be modified without destroying the whole structure. The tangle model is falsifiable: if just one conclusion or just one prediction drawn from the strand conjecture is wrong, it must be abandoned.

So far, experiments in QED are not able to approach Planck scales. This implies that the proposed tangle structure of particles cannot be tested directly. However, indirect tests are possible. Not only is the lack of measurable deviations of any kind from the standard model predicted; at the same time, the lack of any trans-Planckian effects is predicted. Comparison with the perturbative 
expansion of the g-factor appears possible. Observations in the non-perturbative domain of QED - and QCD - might allow further tests in the future.

At present, the strictest tests for the strand conjecture seem to be ab initio calculations of the particle masses, the fine structure constant and the other constants of the standard model. The estimates proposed in this work are not yet precise enough to allow definite statements in favour or against the conjecture. Nevertheless, the strand conjecture is one of the few proposals promising to predict the neutrino mass values before their actual measurement. This work aims at encouraging more research on calculating the frequency of the belt trick that is induced spontaneously by strand fluctuations, and on calculating the probability of photon absorption by an electric charge. In the case that future, more precise calculations disagree with experiments, the strand conjecture would be falsified. If, instead, the calculations agree with experiments, the origin of the colours observed in nature would finally be explained.

\section{Acknowledgments and declarations}

The author thanks Eric Rawdon for his ropelength calculations. The author also thanks Martin Haft, Thomas Racey, David Hestenes, David Broadhurst, Volodimir Simulik, Jason Hise, Claus Ernst, Yuanan Diao, Louis Kauffman, Tyler Spaeth, Jason Cantarella, Marcus Platzer, Antonio Martos, Ralf Metzler, Andrzej Stasiak, Franz Aichinger, Britta Schiller, Sebastian Meyer, Pierre Lacombe and an anonymous referee for discussions and support. Part of this work was supported by a grant of the Klaus Tschira Foundation. There are no additional data available for this work. The author declares that he has no conflict of interest and no competing interests.

\section{References}

[1] H. Kragh, Magic number: a partial history of the fine-structure constant, Archive for History of Exact Sciences 57 (2003) 395-431.

[2] A.O. Barut, The Creation of a Photon: A Heuristic Calculation of Planck's Constant $\hbar$ or the Fine Structure Constant $\alpha$, Zeitschrift für Naturforschung 33a (1978) 993-994.

[3] U.D. Jentschura and I. Nandori, Attempts at a determination of the fine-structure constant from first principles: a brief historical overview, European Physical Journal H 39 (2014) 591-613.

[4] S.L. Glashow and D.V. Nanopoulos, An estimate of the fine structure constant, Nature 281 (1979) 464-465.

[5] M. Shifman, Musings on the current status of HEP, preprint at www.arxiv.org/abs/2001. 00101.

[6] C. Schiller, A conjecture on deducing general relativity and the standard model with its fundamental constants from rational tangles of strands, Physics of Particles and Nuclei $\mathbf{5 0}$ (2019) 259-299. 
[7] C. Schiller, Testing a conjecture on the origin of the standard model, European Physical Journal Plus 136 (2021) 79.

[8] C. Schiller, Testing a conjecture on the origin of space, gravity and mass, Indian Journal of Physics (2021), https://doi.org/10.1007/s12648-021-02209-8.

[9] N. Bohr, Atomtheorie und Naturbeschreibung, Springer (1931).

[10] M. Gardner, Riddles of the Sphinx and Other Mathematical Puzzle Tales, Mathematical Association of America (1987), page 47.

[11] E. Battey-Pratt and T. Racey, Geometric model for fundamental particles, International Journal of Theoretical Physics 19 (1980) 437-475.

[12] V. de Sabbata and C. Sivaram, On limiting field strengths in gravitation, Foundations of Physics Letters 6 (1993) 561-570.

[13] G.W. Gibbons, The maximum tension principle in general relativity, Foundations of Physics 32 (2002) 1891-1901.

[14] C. Schiller, General relativity and cosmology derived from principle of maximum power or force, International Journal of Theoretical Physics 44 (2005) 1629-1647.

[15] C. Schiller, Comment on "Maximum force and cosmic censorship", Physical Review D 104 (2021) 068501.

[16] C. Schiller, Test for maximum force and maximum power, Physical Review D 104 (2021) 124079.

[17] O. Krötenheerdt and S. Veit, Zur Theorie massiver Knoten, Beiträge zur Algebra und Geometrie 5 (1976) 61-74.

[18] J. Cantarella, R.B. Kusner and J.M. Sullivan, On the minimum ropelength of knots and links, Inventiones Mathematicae 150 (2002) 257-286.

[19] A. Martos, Dirac's belt trick for spin 1/2 particle, video at www.vimeo.com/62228139, and A. Martos, Belt trick for the exchange of two fermions, video at www.vimeo.com/62143283.

[20] R.P. Feynman, QED - The Strange Theory of Light and Matter, Princeton University Press (1988), pages $85 \mathrm{ff}$.

[21] D. Hestenes, Zitterbewegung in Quantum Mechanics, Foundations of Physics 40 (2010) 154.

[22] D. Hestenes, Quantum mechanics of the electron particle-clock, preprint at www.arxiv.org/ abs/1910.10478, and references therein.

[23] D. Hestenes, Zitterbewegung structure in electrons and photons, preprint at www.arxiv.org/ abs/1910.11085, and references therein.

[24] V. Fock and D. Iwanenko, Über eine mögliche geometrische Deutung der relativistischen Quantentheorie, Zeitschrift für Physik 54 (1929) 798-802. 
[25] V. Simulik and I. Krivsky, Once more on the derivation of the Dirac equation, preprint at www.arxiv.org/abs/1309.0573, and references therein.

[26] I.Yu. Krivsky, V.M. Simulik, I.L. Lamer and T.M. Zajac, The Dirac equation as the consequence of the quantum-mechanical spin 1/2 doublet model, TWMS Journal of Applied Engineering and Mathethematics 3 (2013) 62-74.

[27] V.M. Simulik, Relativistic quantum mechanics and field theory of arbitrary spin, Nova Science (2020), chapter 2.

[28] C. Schiller, The Strand Model - A Speculation on Unification, MotionMountain.net (2009), chapters 8 to 12 .

[29] L. Lerner, Derivation of the Dirac equation from a relativistic representation of spin, European Journal of Physics 17 (1996) 172-175.

[30] A.O. Barut, Brief History and Recent Developments in Electron Theory and Quantumelectrodynamics, in D. Hestenes and A. Weingartshofer (editors), The Electron - Fundamental Theories of Physics, Springer (1991) pages 105-148.

[31] K. Reidemeister, Elementare Begründung der Knotentheorie, Abhandlungen aus dem Mathematischen Seminar der Universität Hamburg 5 (1926) 24-32.

[32] R. Boudet, Quantum Mechanics in the Geometry of Space-Time, Springer (2011).

[33] J. Davighi, B. Gripaios and N. Lohitsiri, Global anomalies in the Standard Model(s) and Beyond, preprint at www.arxiv.org/abs/1910.11277.

[34] J.A. Heras, Can Maxwell's equations be obtained from the continuity equation?, American Journal of Physics 75 (2007) 652-657.

[35] L. Burns, Maxwell's equations are universal for locally conserved quantities, Advances in Applied Clifford Algebras 29 (2019) 62.

[36] W.E. Baylis, Surprising symmetries in relativistic charge dynamics, preprint at www.arxiv. org/abs/physics/0410197, and references therein.

[37] W.E. Baylis, R. Cabrera and J.D. Keselica, Quantum/classical interface: classical geometric origin of fermion spin, Advances in Applied Clifford Algebras 20 (2010) 517-545.

[38] ACME Collaboration: V. Andreev et al., Improved limit on the electric dipole moment of the electron, Nature 562 (2018) 355-360.

[39] L. Morel, Z. Yao, P. Cladé and S. Guellati-Khélifa, Determination of the fine-structure constant with an accuracy of 81 parts per trillion, Nature 588 (2020) 61-65.

[40] J. Cantarella, J.H.G. Fu, R. Kusner, J.M. Sullivan and N.C. Wrinkle, Criticality for the Gehring link problem, Geometry \& Topology 10 (2006) 2055-2115, preprint at www.arxiv. org/abs/math/0402212.

[41] D. Hanneke, S. Fogwell Hoogerheide and G. Gabrielse, Cavity control of a single-electron quantum cyclotron: measuring the electron magnetic moment, Physical Review A 83 (2011) 052122. 
[42] T. Aoyama, T. Kinoshita and M. Nio, Revised and improved value of the QED tenth-order electron anomalous magnetic moment, Physical Review D 97 (2018) 036001.

[43] H.C. Ohanian, What is spin?, American Journal of Physics 54 (1986) 500-505.

[44] C.T. Sebens, How electrons spin, Studies in History and Philosophy of Science Part B: Studies in History and Philosophy of Modern Physics 68 (2019) 40-50, preprint at www.arxiv. org/abs/1806.01121.

[45] S. Mele, Physics of W bosons at LEP, Physics Reports 403-404 (2004) 255-270, preprint at www.arxiv.org/abs/hep-ex/0407042.

[46] R.M. Wald, Black hole in a uniform magnetic field, Physical Review D 10 (1974) 1680-1685.

[47] B.R. Holstein, How large is the "natural" magnetic moment?, American Journal of Physics 74 (2006) 1104-1111.

[48] R. P. Feynman, in R. Stoops (ed.), The Quantum Theory of Fields, Proceedings of the Twelfth Solvay Conference on Physics at the University of Brussels, October 1961, Interscience (1961), pages 75-76.

[49] A. Petermann, Fourth order magnetic moment of the electron, Helvetica Physic Acta 30 (1957) 407-408.

[50] D.J. Broadhurst and D. Kreimer, Association of multiple zeta values with positive knots via Feynman diagrams up to 9 loops, Physics Letters B 393 (1997) 403-412.

[51] D. Kreimer, How useful can knot and number theory be for loop calculations?, Acta Physica Polonica B 29 (1998) 2865-2873, preprint at www.arxiv.org/abs/hep-th/9807125.

[52] S. Laporta and E. Remiddi, The analytical value of the electron $(g-2)$ at order $\alpha^{3}$ in QED, Physics Letters B 379 (1996) 283-291, preprint at www.arxiv.org/abs/hep-ph/9602417.

[53] B. Abi et al. (Muon g-2 Collaboration), Measurement of the Positive Muon Anomalous Magnetic Moment to 0.46 ppm, Physics Review Letters 126 (2021) 141801, preprint at www. arxiv.org/abs/2104.03281.

[54] S. Borsanyi, Z. Fodor, J.N. Guenther et al., Leading hadronic contribution to the muon magnetic moment from lattice $Q C D$, Nature 593 (2021) 51-55, preprint at www.arxiv.org/abs/ 2002.12347.

[55] V.A. Berezin, Markov's maximon and quantum black holes, Physics of Particles and Nuclei 29 (1998) 274-277.

[56] Z.-Z. Xing, H. Zhang and S. Zhou, Impacts of the Higgs mass on vacuum stability, running fermion masses, and two-body Higgs decays, Physical Review D 86 (2012) 013013, preprint at www.arxiv.org/abs/1112.3112.

[57] V. Katritch, J. Bednar, D. Michoud, R.G. Sharein, J. Dubochet and A. Stasiak, Geometry and physics of knots, Nature 384 (1996) 142-145.

[58] M Aker et al., First direct neutrino-mass measurement with sub-eV sensitivity, preprint at www.arxiv.org/abs/2105.08533. 
[59] J. Hise, animation available at www.youtube.com/watch?v=DHFdBWU36eY.

[60] C. Yu, H. Schumacher and K. Crane, Repulsive Curves, ACM Transaction on Graphics 40 (2021) 2, 1-21, preprint at www.arxiv.org/abs/2006.07859. 